\title{
Product Development Teams Formation: Effects of Organizational and Product Related Factors
}

\author{
Mohsen Jafari Songhori \\ Department of Computer Science, School of Computing, Tokyo Institute of Technology. mj2417@gmail.com \\ Takao Terano \\ Department of Computer Science, School of Computing, Tokyo Institute of Technology. terano@dis.titech.ac.jp
}

\begin{abstract}
While teams are an essential means for developing products in organizations, how to assemble product development teams remains largely unexplored. According to the social network research, teams might be arranged in either closely connected or sparse structures. Conceptualizing a product development project as collective problem-solving endeavour, we develop a computational model of these projects in which a number of designers, who are arranged in teams with a particular structure, conduct search over an NK(C) performance landscape. Simulation of this model enables us to compare the design performance of teams with either closely connected or sparse structures. Our results indicate that how effectively organizations can integrate design solutions determines which of these two structures results in higher development performance. In addition, with our simulation model, design performance of strategies that employ both closely connected and sparse teams, is contrasted with that of those policies which solely use either of these structures. We find that, regardless of product development project integration capability, strategies that simultaneously utilize both closely connected and sparse teams are likely to achieve higher development performance than the other strategies that only use teams with one particular structure. We discuss the implications of our findings in managing product development project.
\end{abstract}

Key words: Product Development Projects, NK(C) Landscape, Team Structure, Team Assembly

\section{Introduction}

Teams are essential for problem-solving and generation of creative ideas in organizations (Thompson 2011, Liang et al. 2010). The way Product Development (PD) projects use teams highly depends on its final product design features. When developing a relatively simple and bounded product, organizing a small team possibly suffices (Fujimoto 1991, Eppinger et al. 1994). However, nowadays, new products are growing in complexity. As a result, PD projects tend to use large number of PD teams. For instance, over 250 teams were required to develop Boeing 777 (Sabbagh 1996). In another similar practice, Ford company used a high number of teams for its Taurus development (Quinn and Pacquette 1988). 
Performance a system with a number of teams can be significantly affected by its structure (Allen 1977, Guimera et al. 2005). Relatedly, two social structures have been studied in the social network field (Kilduff and Brass 2010). In the closely connected structure (i.e. closure network), a focal actor is surrounded by cohesive social network, whereas, in a sparse structure (i.e. brokerage), an individual is surrounded by the other members belonging to disconnected social groups (Coleman 1990, Burt 1992, 2005). Generally, each of the two structures provides distinct performance advantages. Within closely connected structure, the shared third parties create indirect benefits such as induced commitment and trust (Uzzi 1997, Reagans and McEvily 2003), however, sparse networks yield information advantage by providing diverse information (Burt 2004).

In the PD context, team formation can be quite challenging. Often, PD process includes decomposition and definition of subsystems, and then integration of the developed subsystems (Parraguez et al. 2015). Thus, PD teams engage in an essentially complex development process. Moreover, not all subsystems in a product architecture are of equal importance (Ulrich 1995, Baldwin et al. 2014), and there are core subsystems that are tightly coupled to other subsystems, whereas peripheral ones tend to possess only loose connections to other subsystems (Tushman and Peter 1998). Hence, there can be varying interaction intensity among different teams.

Despite the challenges that team formation pose for PD manager, little research has been conducted on this regard (Sosa and Marle 2013). We aim to fill this gap by investigating the performance effects of adopting, uniquely or combinatively, the following team formation strategies: closely connected teams what we refer to as communicative teams, and sparsely arranged teams that we label as diverse teams.

We conceptualize PD endeavor as collective problem solving. That is, designers are simultaneously solving a set of interrelated problems (Thomke 1997). In particular, development process is conceptualized as collective search over an NK(C) landscape (Kauffman 1993, Lazer and Friedman 2007). In other words, we assume there are $n_{s}$ subsystems, each being developed by one team. Each of the subsystems also has $n_{e}$ design elements that form a system with $N=n_{s} n_{e}$ design elements. Parameters $\mathrm{K}$ and $\mathrm{C}$ represent the average number of interactions between design elements of a subsystem, and different interdependent subsystems, respectively. Moreover, there are $n_{q}$ designers in the team who engaged in either myopically improving their own design task or attending to design interactions (Mihm et al. 2003).

In order to differentiate between PD systems with high and low integration capability, we use the best and average quality performance measures. That is, after simulating search process for a fixed time, we examine either the fitness of the best found design solution, or alternatively, the average fitness of all designers' solutions. These measures bold a critical aspect of product development projects where workers in teams need to integrate the members' knowledge in an ongoing process 
of mutual adjustment as their work progresses (Kozlowski et al. 1999, Gardner et al. 2012). We also consider two other important features of PD projects: product architecture and complexity level of its design space. On the former feature, we consider three different architectures, modular, cyclical and acyclical, that have been observed empirically (Baldwin and Clark 2000, MacCormack et al. 2006, Baldwin et al. 2014). Regarding the latter aspect, we examine team assembly policies for PD projects with different complexity levels as products' design spaces can have varying complexity levels (Fleming and Sorenson 2002).

Our goal is to study performance implications of team assembly strategies. In this line, we define our first research question as: [1] whether PD managers should build teams with closely connected structure (i.e. communicative teams), or those with sparse arrangement (i.e. diverse teams)? Arguments on consequences of spare networks with bridging ties and cohesive structures with strong ties hypothesize the former to be capable of implementing innovative ideas while characterize the latter competent in generating ideas (Obstfeld 2005, Tiwana 2008). Our results also show that the answer to this question is conditional on integration capability of PD project. When design solutions can be discerned at low integration costs, managers should build diverse teams with sparse structure. However, if PD integration process is highly inefficient, using communicative teams with cohesive structure can result in higher performance.

PD managers may consider combinative strategies where teams with both cohesive and sparse structures are employed (e.g. some communicative teams develop a subset of subsystems, while the rest of subsystems are being developed by diverse teams). While these strategies might be more challenging for managers than use of only communicative or diverse teams, whether the former ones result in higher performance than that of the latter ones, should be addressed. In this regard, we seek to address our second research question: [2] whether PD managers applying teams with heterogeneous structure (i.e. having both communicative and diverse teams) achieve higher performance than teams with uniform structure (i.e. using only closely connected teams or teams with sparse arrangement)?

Empirical observations confirm that having both strong ties (that exist in cohesive and closely connected network) and weak ties (which are present in sparse structures) are necessary for creativity and innovation (Capaldo 2007, Tiwana 2008, Zhou et al. 2009, Schultz and Schreyogg 2013). Our result resonate this by expecting higher performance levels for PD projects that employ from both diverse and communicative teams. Furthermore, this implication holds for all PD systems, regardless of their integration capability. This is in line with arguments that expect small world structure to foster creativity (Fleming and Marx 2006) or prescribe "ambidextrous" structure for organizations (O`Reilly and Tushman 2004).

In summary, our results yield several managerial insights on team assembly in PD projects. Whether communicative teams with cohesive structure or diverse teams with sparse arrangement 
should be used, depends on integration capability of project organization. If the best design solutions can be discerned properly, diverse teams appear more promising to result in higher PD performance. Moreover, if integration and identification of best design solutions is substantially challenging, then use of communicative teams, rather than diverse teams, engenders a high PD performance. Lastly, our model expects higher performance levels for combination of both communicative and diverse teams than building teams with uniform social arrangement (e.g. communicative teams).

The paper proceeds as follows. Section 2 presents a review of the relevant literature. The subsequent section describes the mathematical model of designers' collective search effort. These explanations are followed by details of PD performance measures and team formation strategies. In Section 4, we elucidate our simulation experiments and report the results. Finally, Section 5 discusses implications and limitations of the results, and concludes the paper.

\section{Literature Review}

\subsection{Team Assembly in Product Development Projects}

The central debate on the structural dimension of social capital revolves around whether more informational and performance benefits are gained from closely connected (i.e. closure) networks or sparse (i.e. brokerage) structures (Kilduff and Brass 2010). In the closely connected network, a focal actor is surrounded by cohesive social network, whereas, in the latter, a focal actor is surrounded by the other actors belonging to disconnected social groups (Coleman 1990, Burt 1992, 2005). Intuitively, each of these network types provides distinct performance enhancement. Within closely connected structure, the shared third parties create indirect benefits such as induced commitment and trust (Uzzi 1997, Reagans and McEvily 2003), however, sparse networks yield information advantage by providing diverse information (Burt 2004).

Closely connected teams structure provides a supportive environment that is more desirable for innovation (Obstfeld 2005). Such common third-parties also motive the designers to engage in design-related interactions, and consequently, they create a collaborative atmosphere that is needed for creativity (Coleman 1990, Obstfeld 2005). Dense structure and third-parties provide trust, and increase information sharing among designers. With such information sharing behavior, teams are likely to have higher performance. This argument has also been echoed in some discussion on R\&D teams arrangement. Especially, decreasing spatial distance of these teams is expected to improve PD performance by improving communication frequency among those teams (Allen 1977, Krishnan and Ulrich 2001, Bardhan et al. 2013). However, high communication levels among PD teams may not always improve PD performance. For instance, while cyclic interdependencies among designers are common in PD projects (Smith and Eppinger 1997), managing those communicative interactions can be difficult, and they can increase design errors occurrences (Sosa et al. 2013). 
In a sparse social network, when a focal designer has interactions with a large number of other designers that tend to be disconnected from each other (i.e. a sparse network is present around the focal designer), the focal designer is more likely to be creative and innovative. Essentially, this association drives from the fact that a sparse network most probably gives the focal actor access to diverse knowledge, which can be combined into novel outcomes (Burt 2004, Fleming et al. 2007). In addition to the structural features, knowledge-diversity content of designers interactions has been considered to improve creativity conditions for design teams (Sosa 2011).

In the PD literature, the discussed cohesive and sparse social structures are also found to be associated with distinct teams performance advantages. For instance, in the study of a software development company, Sosa (2011) analyzed engineers' interactions, and found dyadic interactions that channel various knowledge domains are more likely to facilitate generation of creative ideas. That study also observed network cohesion (i.e. common third parties) below average levels, support generation of creative ideas, and highly cohesive social network of engineers can hinder creativity. By examining other aspects of the same software development data, Sosa (2014) observed positive effects of a social embeddedness of designers' dyadic relationship - combination of tie strength and network cohesion - on the probability that they realize need for design rework (i.e. corrective or completion actions).

Despite usefulness of the previously discussed insights, PD teams formation can be quite challenging due to the essential complexity in PD systems. On the one hand, usually, the process of developing a complex product includes decomposition and definition of subsystems (i.e. conceptual \& system level design in (Parraguez et al. 2015)), and then integration of the developed subsystems. Each subsystem represents a subset of the product architecture which includes a group of components that collectively perform a higher level function (Gokpinar et al. 2013). For instance, in a vehicle development project, subsystems such as front suspension, steering wheel, door trim, front seat, etc. may be defined. Similarly, a group of source files that are related to a part of software design can be defined as software subsystem (MacCormack et al. 2006).

On the other hand, according to the product architecture concept, not all subsystems in a product architecture are of equal importance (Ulrich 1995, Baldwin et al. 2014). All complex systems, including PD systems, can be described in terms of their architecture - that is, as a hierarchy of subsystems that in turn have their own subsystems (Simon 1962). Product architecture has been defined as " the scheme by which the function of a product is allocated to its constituent components " (Ulrich 1995). From architecture perspective, some subsystems are "core" to system performance, whereas others are only "periphera" (Tushman and Rosenkopf 1992). Core subsystems have been defined as those that are tightly coupled to other subsystems, whereas peripheral subsystems tend to possess only loose connections to other subsystems (Tushman and Peter 1998). Such core-peripheral 
structure has been found in development of complex products (MacCormack et al. 2006, Baldwin et al. 2014).

\subsection{Product Development Integration}

Knowledge in projects are complex, and consequently, in a distributed environment like projects, organizational routines and norms often interrupted over the course of project. Relevantly, work integration has been defined as mechanisms across all organization and task boundaries that maintain the integrity of the distributed project work from the beginning until the end (Anderson and Parker 2013). On this regard, we define organizational types with high and low integration capabilities. The former ones can integrate different design solutions proposed by team members is likely to develop a product using the best design solutions for each subsystem with low costs. Contrarily, the latter ones are more likely to converge toward lower quality designs.

Our differentiation between highly and lowly capable PD organizations is in line with the fact that workers in teams need to integrate the members' knowledge in an ongoing process of mutual adjustment as their work progresses (Kozlowski et al. 1999, Gardner et al. 2012). The knowledge integration, in other words, makes some teams more effective than other teams by developing dynamic capabilities that enable the former teams to integrate knowledge in a systematic and reliable way (Gardner et al. 2012).

Distinguishing the highly capable projects from the ones with low capability also seems plausible from the perspective of operational and product performance measures. Usually, the former is related to project goals such as adherence to schedule and budget, while the latter examines financial and market performance of an innovative project (Blindenbach-Driessen et al. 2010, Tatikonda and Montoya-Weiss 2001). In addition, although operational performance is generally considered to improve product performance (Tatikonda and Montoya-Weiss 2001), that may not be valid for all firms (Griffin and Page 1996, Ancona and Bresman 2013). According to this view, highly capable projects can be seen as those projects that achieve high operational and product performance levels by identifying and integrating best design solutions. However, the low capable PD systems may be conceptualized as the ones with low operational and product performance levels, as they reach only to the average solutions.

Different integration capability levels of PD projects can be resulted from their organizational context and environment. Generally, organizational context in which teams operate affect their performance by providing them access to technical knowledge and resources to solve their problems (Griffith et al. 2003). In addition, some teams may have access to more resources or face less constraint from their organizational context (Young-Hyman 2016). Organizational context should create environment where top manager translate prioritized strategic themes into actionable goals 
for middle management, while the latter coordinate with each other and report upwards on the progress of implementation to enable corrective actions (Ethiraj and Levinthal 2004, Vuori and Huy 2015). Hence, interactions among top and middle managers are important part of organizational context for innovation success. Nevertheless, this can be challenging given that individuals may tend to avoid bringing up issues during their interactions (Vuori and Huy 2015). Such poor mutual understanding between top and middle managers in the innovation process (Tripsas and Gavetti 2000, Gilbert 2005), and its consequences for Nokia's recent innovation challenges (Vuori and Huy 2015) can be found in the literature.

\subsection{Interaction Pattern and Collective Problem Solving Perspective}

The PD literature admits that a product's architecture which maps functions of a product to its components (Ulrich 1995) is not unique, and, there could be more than one architectures that satisfy the functional requirements of a product (MacCormack et al. 2006). Such architectures probably differ along various performance attributes like product quality and reliability (Ulrich 1995), partitioning of design tasks and efficiency of development process (Von Hippel 1990). Understanding how such product architectures make performance trade-offs has been critical topic for scholars (MacCormack et al. 2006). Following this line of research, we examine how different product architectures affect performance of various team formation strategies.

In a broader context than PD, empirical work in organizational, social, and technological domains has shown that interactions among elements of these systems are patterned, rather than random (see Rivkin and Siggelkow (2007)). Additionally, interaction patterns such as modular, hierarchical, and small-world have been considered (Watts 1999, Rivkin and Siggelkow 2007, Baldwin and Clark 2000). Focusing on PD context, we use the following patterns that are shown in Table 1 for five teams. Cyclical (or centralized) pattern is a concept that is known as "core-periphery" in some studies of development projects. Also, what we refer to as acyclical pattern is also known as hierarchical pattern (MacCormack et al. 2006, 2012, Baldwin et al. 2014). These two patterns capture the extent to which each subsystem depends on itself via other subsystems (Sosa et al. 2013). In a cyclical pattern, there is a high degree of cyclicality and one team's design decisions affect its own search landscape also via its impact on other teams' search landscape. This phenomenon is absent in an acyclical subsystem interaction pattern. We also include modularity pattern as it has been an important feature of product architectures (Ethiraj and Levinthal 2004, Ethiraj et al. 2008).

Development of a new product often is conducted in a distributed manner. In such a system, teams of designers simultaneously make partially autonomous decisions about product's components that affect the overall complex product performance (Mihm et al. 2003). Such description of PD is consistent with PD conceptualization as decision-makers collectively solve a distributed information 
Table 1 Interaction network among five teams

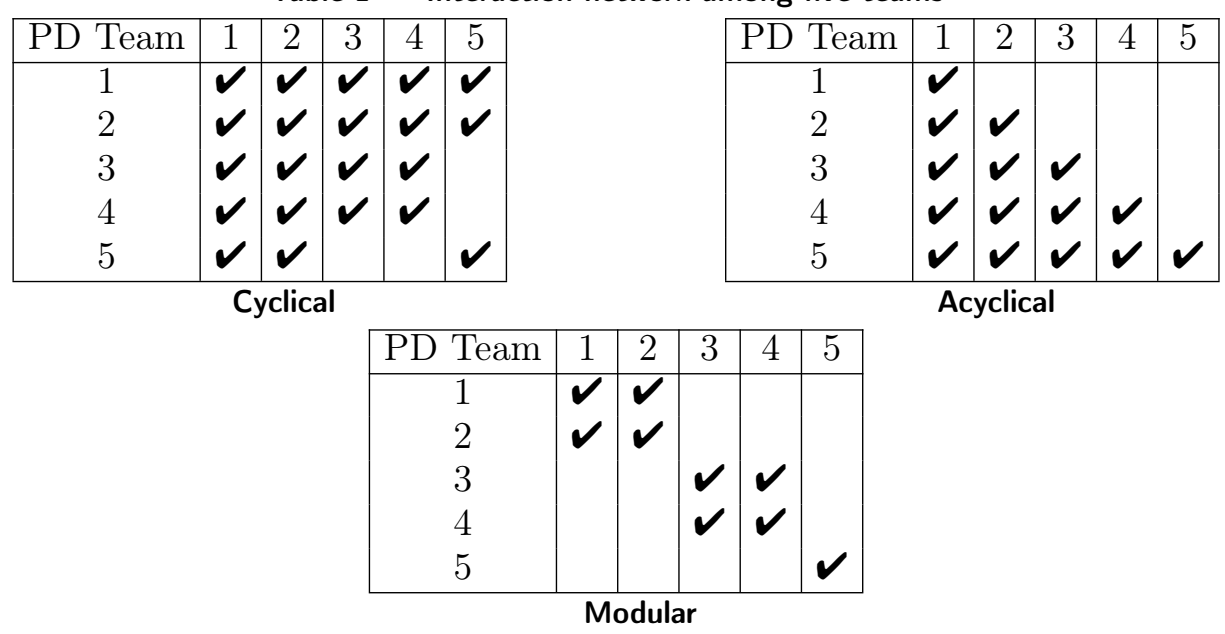

processing problem (Thomke 1997). During the design process, designers engage in locally optimizing features of a component. In addition, due to interactions between components, designers need to have an ongoing communication process (Mihm et al. 2003). The explained PD process can be pictured as collective problem solving in PD organization. In particular, PD projects can be seen as several specialists solving an innovative search problem (Knudsen and Srikanth 2014). Here, not only an engineer's own problem-solving (i.e. design) activities, but also activities of the other peers can affect that engineer's design activity.

We conceptualize distributed PD project as collective problem solving effort by some agents (i.e. designers) for a complex problem. Collective problem solving efforts by interconnected agents have been studied in the literature of complexity and organizational decision-making (Barkoczi et al. 2015). For instance, Lazer and Friedman (2007) used an extended NK landscape model to show how network structure can affect the resulting collective problem-solving attempt. As another example, contemplating interpersonal network in organizations, and using a computational model, Fang et al. (Fang et al. 2010) illustrated that when subdivided into semi-isolated subgroups, the organization's long-term learning performance is expected to be significantly higher than when the network is not divided into subgroups or when subgroups have many cross-group links.

\section{Model}

To understand how team formation strategies affect PD performance, we build an Agent-Based simulation Model (ABM) of PD that is an extension of the well-studied NK landscape (Kauffman 1993). In this section, we set up the mathematical model used to simulate the search process. The model constituents are (I) characterization of the landscape over which the teams conduct search, (II) organizational arrangement based on which designers interact, and teams interaction strength measure, (III) the collective search endeavor by designers, (IV) the defined performance measures, and finally, (V) the applied team-formation strategies. 


\subsection{The Landscape Model}

Consider a product with $n_{s}$ subsystems which are represented by $S_{s}=\left\{i \mid 1,2, \ldots, n_{s}\right\}$. We model the design landscape of the product using the NK landscape model (Kauffman 1993). In this terminology, the landscape of subsystem $i$ consists of $n_{e}$ interacting binary elements that are in either state 0 or state 1 at any given time. The total number of elements for $n_{s}$ subsystems is thus $N=n_{e} n_{s}{ }^{1}$ The state of subsystem $i$, denoted by $s^{i}$ is known when the states of its $n_{e}$ elements are known; that is, $s^{i}=\left\{e^{i x} \mid e^{i x} \in\{0,1\}, x=1,2, \ldots, n_{e}\right\}$. Consequently, each subsystem has $2^{n_{e}}$ different design states. For example, the state of subsystem $i$ when $n_{e}=5$ might be (00110). Similarly, and from a higher level perspective, the product state denoted by $\mathbf{S}=\left\{\mathbf{s}_{\mathbf{r}}=\cup_{i=1}^{i=n_{s}} s^{i} \mid \mathbf{r}=1,2, \ldots, 2^{N}\right\}$ can have $2^{N}$ possible states.

For each of $2^{N}$ possible states (i.e. design alternatives), there is a defined product performance. This performance is represented by a fitness value, and defined as the average of contributions of the product subsystems' elements. The contribution of each element $e^{i x}$ is defined such that it depends on the following factors: (i) the state of the element $e^{i x}$ itself, (ii) the state of $K$ other randomly-selected elements of subsystem $i$ that interact with element $e^{i x}$ and (iii) the status of $C$ randomly-selected elements of the other subsystems that interact with that subsystem of element $e^{i x}$. The second ingredient captures the idea that the elements under teams' control are interdependent; in particular, the contribution of one element depends on the status of $K$ other elements. The third ingredient materializes the idea that subsystems are interdependent and so contribution of element $e^{i x}$ depends on the $C$ elements of each subsystems that interacts with subsystem $i$. Denote the set of subsystems interacting with subsystem $i$ as $\mathrm{DE}_{i}$. For instance, if subsystem $i$ interacts with three other subsystems, then the contribution of $e^{i x}$ depends on $K+3 \times C$ other elements.

For the first stage of NK model, the interaction patterns among the subsystem's elements should be determined. As discussed earlier in Section 2.3 (see Table 1), different interaction patterns, e.g. modular, cyclical, have been observed in the empirical studies, and are used in this paper.

The second stage of the NK model involves the landscape function generation; that is to generate the contributions of the subsystem elements. More precisely, as there are $K+\left|\mathrm{DE}_{i}\right| \times C$ interacting elements with element $e^{i x}$, the number of required contributions for this element are $2^{K+\left|\mathrm{DE}_{i}\right| \times C}$. These contribution values are drawn from a uniform $[0,1]$ distribution. It has been shown that the properties of the fitness landscape are not sensitive to the distribution applied to generate the landscape (Weinberger 1991).

Once the product elements' contributions at all possible states (i.e. design alternatives) are generated, we can construct the product landscape (i.e. the product performance for each possible design

\footnotetext{
${ }^{1}$ We assume that all subsystems have the same number of elements $n_{e}$.
} 
alternative is calculated). Let $f\left(e^{i x} \mid \mathbf{s}_{\mathbf{r}}\right)$ be the generated contribution of element $e^{i x}$ when product state is $\mathbf{s}_{\mathbf{r}}$; Therefore, product performance at state $\mathbf{s}_{\mathbf{r}}$, is represented by a fitness value, $f\left(\mathbf{s}_{\mathbf{r}}\right)$, which is the average of the contributions of its subsystems' elements:

$$
f\left(\mathbf{s}_{\mathbf{r}}\right)=\frac{\sum_{x=1}^{n_{e}} f^{i}\left(e^{i x} \mid \mathbf{s}_{\mathbf{r}}\right)}{n_{e}}
$$

The third, and last, stage in the NK model is characterization of the search process. This will be described in the next sections.

\subsection{Organizational Structure and Teams Interaction Strength}

We generate organizational network or team arrangement using the "connected caveman" model, that was developed by (Watts 1999), and recently used by (Fang et al. 2010) to create interpersonal organizational structure. In that network representation of PD project, nodes (i.e. agents) correspond to the designers in teams, and clusters represent teams. Hence, designers of each PD team are highly clustered and minimally connected to the other PD teams. Also, to materialize various social structures in this model, designers' absorptive capacity (i.e. capability to understand shared design solutions by the other designers) as well as the designers' expertise similarity are varied across teams (more details are provided in Section 3.4 in below). Therefore, different team formation strategies are considered to affect designers' absorptive capacity and their expertise distribution, who are collectively conducting search over a performance landscape (see Figure 1 in below).
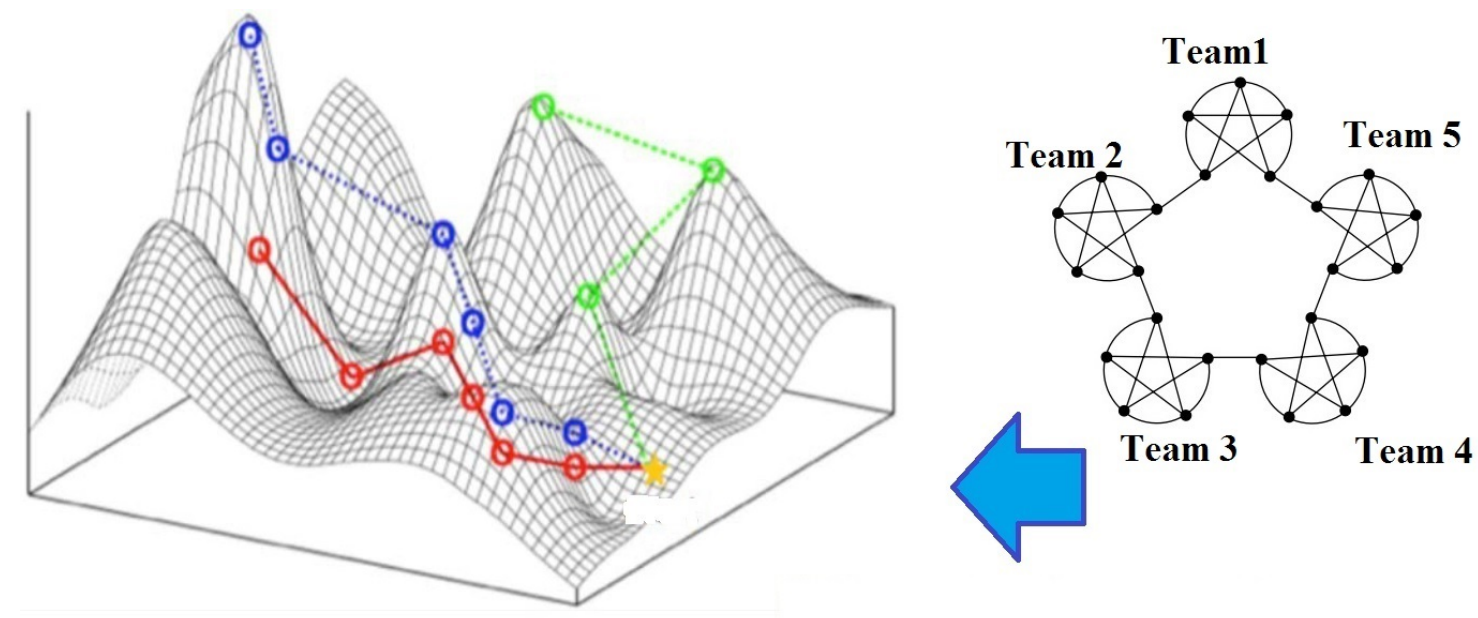

Figure 1 Caveman network (Watts 1999, Fang et al. 2010) for PD teams organizational arrangement (right) that affects collective search effort over design landscape (left).

In our model, designers in the design teams conduct the search process over landscape of subsystems. Assume there are $n_{s}$ teams, denoted by $\mathbf{D}$, and each team $j$ is developing subsystem $j$. 
Moreover, each team $j$ is comprised of $n_{q}$ number of designers, $D_{j}=\left\{d_{q}^{j}, q=1,2, . ., n_{q}\right\}$. We assume operation of a fixed number of teams for development of each subsystem. Let's define the subset of

designers $\mathbf{D}_{\mathbf{q}}^{\mathbf{j}} \subset \mathbf{D}$ to be designers $d_{q^{\prime}}^{j^{\prime}}$ who have organizational interactions with designer $d_{q}^{j}$. This set for each designer is determined according to the organizational network structure.

As discussed earlier, each team is developing a particular subsystem with varying interaction and criticality level. To model this, subsystem' interaction intensity with all other subsystems is considered. Toward that end, we define a "subsystem interaction strength" measure that is based on how many subsystems affect the fitness value of subsystem $j$ (subsystem $i=j$ ) that is being developed by team $j$ (i.e., $\left|\mathrm{DE}_{j}\right|$ ), and also, how many subsystems' fitness values are affected by subsystem $j$ that is being developed by team $j$ (i.e., $\left|\mathrm{SI}_{j}\right|$ ). Especially, we define interaction strength measure as follows that captures the overall influence of team $j$ in the space of a complex PD project. In equation $2, W>1$ is a weight parameter by which we differentiate between the interactions by which a focal team influences other teams and those by which it is impacted by other teams. Interaction strength measure $m_{j}$ is similar to the other defined constructs in the literature (i.e. Fan-out and Fan-In measures in (Baldwin et al. 2014)) for the visibility matrix which calculates the extent of redesign iterations/efforts for the changes in a particular element of a system (Sharman and Yassine 2004, MacCormack et al. 2006, Baldwin et al. 2014).

$$
m_{j}=\frac{\left|\mathrm{DE}_{j}\right|+W \times\left|\mathrm{SI}_{j}\right|}{(1+W) n_{s}}
$$

The defined measure enables us to define scenarios in which the teams tend to have particular structure and behaviors. That is, teams are established to have particular features with a probability that is a function of interaction strength measure $m_{j}$ (e.g. increasing or decreasing in $m_{j}$ ).

\subsection{Design Process as Collective Search}

We conceptualize a PD project as parallel collective search process conducted by the designers of PD teams. That is, the design solution of each designer, at any time $t$, is regarded as a position over the product landscape with $N$ decisions (i.e. a design alternative among $2^{N}$ alternatives), and each focal designer conducts his own search while communicating with other designers at some occasions.

In our model, initially and at time $t=0$, each designer $d_{q}^{j}$ is assigned a randomly selected design solution or state $\mathbf{s}_{\mathbf{q}, \mathbf{j}}^{\mathbf{0}}$ and his fitness value is calculated. Moreover, at each subsequent time $t$, each designer $d_{q}^{j}$ engages in either design improvement through local search or communicating design solutions with the other designers (Lazer and Friedman 2007, Lin 2014). At first, at each simulation time, designers engage conducting local search. If the local search endeavor was successful, and a focal designer finds a superior soultion, then he will not perform any other task at time $t$. However, the set of designers whose local search process did not result in a superior solution, get involved in communicating with the other designers who are also active in the latter process at each time $t$. 
According to the local search process, a focal designer changes one of the state of the design elements in his expertise domain, i.e. $e^{i x} \in E X_{q}^{j}$, from 0 to 1 or vice versa. That generates a new alternative, $\tilde{s}_{q, j}^{t+1} \in \mathbf{S}$, which the designer accepts if the result is a higher fitness value; thus,

$$
\mathbf{s}_{\mathbf{q}, \mathbf{j}}^{\mathbf{t}+\mathbf{1}}=\tilde{s}_{t+1}^{q, j} \quad \text { if } \quad f\left(\tilde{s}_{q, j}^{t+1}\right)>f\left(\mathbf{s}_{\mathbf{q}, \mathbf{j}}^{\mathbf{t}}\right) .
$$

Otherwise, designer $d_{q}^{j}$ retains the previous alternative and $\mathbf{s}_{\mathbf{q}, \mathbf{j}}^{\mathbf{t}+\mathbf{1}}=\mathbf{s}_{\mathbf{q}, \mathbf{j}}^{\mathbf{t}}$.

Once all the communicating designers at time $t$ are recognized, we create pairs of communicating designers who have organizational interactions. In doing so, there could be few designers for whom communicating partners can not be found. Those unpaired designers then retain their design solution (i.e. position over performance landscape) for the next time period. Assume designers $d_{q^{\prime}}^{j^{\prime}}$ and $d_{q^{\prime \prime}}^{j^{\prime \prime}}$ are selected for communication at time $t$, and $d_{q^{\prime \prime}}^{j^{\prime \prime}} \in \mathbf{D}_{\mathbf{q}^{\prime}}^{\mathbf{j}^{\prime}}$, and also, assume the latter has higher fitness than the former, i.e. $f\left(\mathbf{s}_{\mathbf{q}^{\prime \prime}, \mathbf{j}^{\prime \prime}}^{\mathbf{t}}\right)>f\left(\mathbf{s}_{\mathbf{q}^{\prime}, \mathbf{j}^{\prime}}^{\mathbf{t}}\right)$. Then, each element of the design solution of designer $d_{q^{\prime \prime}}^{j^{\prime \prime}}$ is imitated with probability $A C_{q^{\prime \prime}, q^{\prime}} \in[0,1]$, and, used by designer $d_{q^{\prime}}^{j^{\prime}}$, at next time period. In

addition, designer $d_{q^{\prime \prime}}^{j^{\prime \prime}}$ retains the current design alternative for the next period, $\mathbf{s}_{\mathbf{q}^{\prime \prime}, \mathbf{j}^{\prime \prime}}^{\mathbf{t}+\mathbf{1}^{\prime \prime}}=\mathbf{s}_{\mathbf{q}^{\prime \prime}, \mathbf{j}^{\prime \prime}}^{\mathbf{t}}$. This process is repeated for all designers who are communicating with each other at time $t$.

The defined parameter $A C q^{\prime \prime} q^{\prime}$ represents designers' absorptive capacity (Cohen and Levinthal 1990), and shows the percentage of the design solution (e.g. decision string $[0,1,0,1]$ ) of designer

$d_{q^{\prime \prime}}^{j^{\prime \prime}}$ that are imitated by agent $d_{q^{\prime}}^{j^{\prime}}$, and is inversely associated with the knowledge and background diversity level in teams (i.e. the highly diverse team has low absorptive capacity where low percentage of design solutions communicated among designers). Cohen and Levinthal (1990) defined absorptive capacity as "... prior related knowledge confers an ability to recognize the value of new information, assimilate it, and apply it to commercial ends.". Since absorptive capacity can be defined at different levels, e.g. individual, team, our defined parameter and modeling assumption appear to be plausible.

When a designer attends to his own design and conducts a local search, his expertise domain can significantly affect the search process effectiveness. Following the former modeling assumptions (Kavadias and Sommer 2009), we assume a focal designer $d_{q}^{j}$ is knowledgeable about a subset of $n_{e}$ design elements of subsystem $i$, that is denoted by $E X_{q}^{j} \subset s^{i}$. Additionally, the expertise domain of each designer is defined to cover only one design element, $\left|E X_{q}^{j}\right|=1 \forall d_{q}^{j}$.

\subsection{Teams with Different Structures}

As discussed earlier, depending on team formation strategies, PD teams with different social structures may be used. Especially, those teams may have closely connected or sparse network structures. In our model, such structures are manifested as different communication effectiveness and expertise distribution within teams. When PD teams have closely connected and cohesive structure, designers are more likely to have higher absorptive capacity, and, also possess similar knowledge domains. 
However, in teams with sparse structure, designers have diverse expertise, and their communication is less likely to be effective.

At first, we consider PD projects where managers apply similar structures for all PD teams. To this end, teams with two different structures are elaborated. In these projects, team features like designers communication effectiveness and expertise distribution are unrelated to the teams' subsystems features like its interaction strength measure (i.e. $m_{j}$ that was defined in Section 3.2). Let $p_{d d}^{y} \in[0,1]$ denote the diversity level that policy $y$ applies for team formation. Then, we assume that team diversity level (i) is adversely related to the probability that designers in teams have similar expertise domain, or mathematically, $P\left(E X_{q}^{j}=E X^{j}\right)=1-p_{d d}^{y}, \forall d_{q}^{j}$ and $\left|E X^{j}\right|=1$, and,(ii) is adversely associated with the designers' absorptive capacity level, or mathematically, $A C_{q^{\prime \prime} q^{\prime}}=$ $1-p_{d d}^{y} \forall d_{q^{\prime \prime}}^{j}, d_{q^{\prime}}^{j}$.

We define communicative teams to be assembled by considering a low diversity level, $p_{d d}^{1} \approx 0$. As a result, the designers' absorptive capacity is high and, also with a high probability designers have similar expertise. Differently, diverse teams, are assembled with sparse structured. Hence, a high diversity level, $p_{d d}^{2} \approx 1$ is enforced. Consequently, designers' absorptive capacity is low and, also with a low probability, designers have similar expertise domains.

To investigate performance of PD projects that simultaneously utilize both communicative and diverse teams, we define two other PD systems. In these PD systems with heterogeneous team structures, designers' absorptive capacity and their likelihood to have similar expertise are associated with teams interaction strength measure $\left(m_{j}\right.$ that was defined in Section 3.2). To that end, we also use core and peripheral concepts that has been defined in the literature (Tushman and Rosenkopf 1992, Tushman and Peter 1998). This enables us to investigate whether performance of PD systems with heterogeneous teams are effected by team structure of those who are developing subsystems with higher/low interactions strength.

We define another type of PD systems where teams with higher interaction strength — who develop more central subsystems - are formed with more closely connected structure. That is, a set of designers who have shared third-parties - or simply they cohesive structure - are allocated to more central subsystems. Put it differently, the more teams have sparse and disconnected structure, the less central/core subsystems they design. Hence, we refer to these teams as "diverse teams developing peripheral". The following assumptions are considered to form diverse teams developing peripheral : (i) probability that designers in team $j$ have similar expertise domain is increasing in their interaction strength, or mathematically, $P\left(E X_{q}^{j}=E X^{j}\right)=m_{j}, \forall d_{q}^{j}$ and $\left|E X^{j}\right|=1$, and, (ii) the designers' absorptive capacity is increasing in their interaction strength: $A C_{q^{\prime \prime} q^{\prime}}=m_{j} \forall d_{q^{\prime \prime}}^{j}, d_{q^{\prime}}^{j}$.

Last type of elaborated PD teams are such that they tend to have more closely connected structures as the subsystems they develop have lower interaction strength. In other words, following this 
strategy, diverse teams develop more core subsystems-that have higher number of interactions, and consequently, we refer those teams as "diverse teams developing core". More precisely, diverse teams developing core are characterized as: (i) probability that designers in team $j$ have similar expertise domain is decreasing in their interaction strength, or mathematically, $P\left(E X_{q}^{j}=E X^{j}\right)=1-m_{j}, \forall d_{q}^{j}$ and $\left|E X^{j}\right|=1$, and, (ii) the designers' absorptive capacity is decreasing in their interaction strength: $A C_{q^{\prime \prime} q^{\prime}}=1-m_{j} \quad \forall d_{q^{\prime \prime}}^{j}, d_{q^{\prime}}^{j}$.

\subsection{Product Development Performance}

As a collective endeavor, design solutions should be acknowledged and accepted by all members of a team, and also, they need managerial approval. Thus, not only discovery of a design solution, but also, its acceptance in the organization needs to be considered. Especially, the latter process requires managerial and organizational resources. This can be discussed from the integration (Anderson and Parker 2013, Mishra and Sinha 2016) perspectives. This aspect emphasizes the fact that knowledge in projects are complex, and consequently, in a distributed environment like projects, organizational routines and norms often interrupted over the course of project. Hence, some mechanisms across organization and task boundaries are necessary to integrate the distributed project work (Anderson and Parker 2013).

To materialize effectiveness of firms in acceptance and integration of a superior solution among a team's designers, we define two quality measures. One measure of product quality uses the best design solution discovered by designers of each team for its subsystem. The second quality measure is the average fitness of all design solutions found by all designers. An organization with high capability of integrating different design solutions proposed by team members is likely to develop a product using the best design solutions for each subsystem with low costs. In other words, firms with high integration capability presumably can apply the former quality scale. However, when PD systems are less capable (e.g. unable to cope with high integration costs), they are more likely to converge toward lower quality designs. In our paper, that lower product design is represented by the second quality scale or the average of design solutions of all designers ${ }^{2}$.

In this paper, the first quality scale that uses the best design solutions for each subsystem is called best quality measure. Let fitness value $Q_{i j}^{t}$ indicate the quality of subsystem $i$ at time $t$ that is being developed by team $j(i=j)$. Then, the overall best product quality at time $t$ is average of subsystem's quality:

$$
P Q^{t}=\frac{\sum_{j=1}^{j=n_{s}} Q_{i j}^{t}}{n_{s}} \quad \text { such that; } \quad Q_{i j}^{t}=\operatorname{Max}_{q}\left[f\left(\mathbf{s}_{\mathbf{q}, \mathbf{j}}^{\mathbf{t}}\right)\right] \quad \forall d_{q}^{j} \in D_{j}
$$

${ }^{2}$ In a very abstract view, the first quality measure represents capability of PD system to analyze all design solutions, and select the best ones found for each subsystem. Whereas, the second quality scale indicates less capable systems that use lower amount of resources, and converge toward the average fitness of all designers' solutions. 
The second quality scale, or average quality, calculates the average fitness of the solutions found by all designers. Thus, the overall product quality can be represented as:

$$
V^{t}=\sum_{j=1}^{j=n_{s}} \sum_{q}\left[f\left(\mathbf{s}_{\mathbf{q}, \mathbf{j}}^{\mathbf{t}}\right)\right] \quad \forall d_{q}^{j} \in D_{j}
$$

\section{Experiments and Results}

In this section, we describe the experimental setup and report the results. One set of parameters in our model are related to the general setting of PD project. The parameter $N$ in the NK simulation model is the total number of elements in the landscape: $N=n_{s} \times n_{e}$. In our experiments, we assume that the product consists of $n_{s}=5$ subsystems and that each subsystem's landscape consists of $n_{e}=4$ interacting elements, respectively; hence $N=n_{s} \times n_{e}=4 \times 5=20$. Furthermore, we assume there are $n_{q}=5,7$ number of designers in each teams. Hence, PD project with five subsystems, includes $5 \times 5=25(5 \times 7=35)$ designers (i.e. agents) in total.

The second type of parameters define the PD landscape features. The ruggedness of search landscape depends on both parameters $K$ and $C$-in other words, on how interdependent the elements of subsystems are (Levinthal 1997). Low values of $K$ and $C$ imply that the contribution of one element is rather limited and independent of the other elements; hence the landscape is relatively smooth and a change in the state of one element does not significantly affect the fitness of others. However, if the values of $K$ and $C$ are high then the fitness landscape becomes more rugged; here a change in the state of one element may have a significant effect on the fitness values of other elements. In such landscapes, an incremental search process may stop at a local optimum.

To cover both smooth and rugged landscapes, we assume parameters $K$ and $C$ to be either 1 or $n_{e}-1$. Consequently, there are four different PD settings: (1) Elements of both a subsystem and different subsystems are intensively interdependent $\left(K=C=n_{e}-1\right),(2)$ Elements of both a subsystem and different subsystems are mildly interdependent $(K=C=1)$, (3) Elements a subsystem are intensely interactive, whereas, elements of different subsystems have low number of interactions ( $\left.K=n_{e}-1, C=1\right)$, and, (4) Elements a subsystem are lightly interactive, whereas, elements of different subsystems are highly interdependent $\left(K=1, C=n_{e}-1\right)$. Among these project types, the one in which elements of both a subsystem and different subsystems mildly interact $(K=C=1)$, has smallest number of interactions among elements, and consequently, its landscape is smooth. However, the other project types have more rugged landscapes as there are higher number of interactions among elements (e.g. $K=1, C=n_{e}-1$ ).

It worths noting that the four defined PD project settings also indicate the architectural knowledge of firm. In other words, interaction intensity within and between subsystems, or $K$ and $C$ respectively, represent the organization effectiveness in managing interactions. When, within and 
between subsystem interactions are light and intense $\left(K=1, C=n_{e}-1\right)$, it means that the elements interactions have not been managed properly - as each team is developing a subsystem that its elements are independent to a large extent. Differently, in the other project types, within subsystem interactions are more intense than, or at least similar to, that of between subsystem interactions. Hence, these interactions among the elements of these products are managed more properly.

Subsystems in a PD project may relate to each other in different architectures (patterns). Table 1 in Section 3.2 shows the three patterns, cyclical, acyclical and modular patterns, that we use in our experiments for $n_{s}=5$ teams ${ }^{3}$.

\subsection{Experiment 1: Uniformly Assembled Teams}

Managers may use the same policy to form teams for all subsystems. In our first set of experiments, we investigate whether closely connected and less diverse teams result in higher PD performance than that of sparse and diverse teams. Will PD projects have superior performance if they use one of these team formation strategies? Also, does the answer to this question depend on either the interaction pattern among product subsystems or the level of interactions intensity? How about integration capability of firm?

We compare the performance of a PD project with two team structures: (i) communicative teams who have high absorptive capacity and similar expertise, (ii) diverse teams whose communication is less effective and have diverse expertise. To that end, we assume the former and latter assign low $p_{d d}^{1}=0.1$ and high $p_{d d}^{2}=0.9$ diversity levels, respectively. We compare these strategies based on both the best and average quality measures that are defined in equations 4 and 5 . We simulate each scenario for a fixed number of time periods and record the performance measures of all teams.

For each combination (PD setting and subsystems' interaction pattern), we generate 100 landscapes. For each landscape, we create the corresponding PD project scenario (e.g. with communicative teams) and let teams conduct the search process for 100 time periods. This process is repeated for each of the 100 landscapes. Then, performance at each simulation time is the average performance at that time period across all landscapes.

Graphs in Figure 2 depict the best quality performance $\left(P Q^{t}\right.$ in equation 4$)$ of $\mathrm{PD}$ projects with $K=C$ setting ${ }^{4}$. According to panels (a)-(d), deploying diverse teams results in higher longterm performance than using communicative teams. This holds for all PD settings with various subsystems interaction pattern, and also, different interaction intensity levels (i.e. within-team, $K$, and between-team $C$ interaction levels). However, across all panels, in short-run, PD systems

\footnotetext{
${ }^{3}$ The patterns for $n_{s}=7$ teams have exactly the same pattern as those shown in Table 1 . Note that modular pattern is perfectly modular with every two teams having interactions only with themselves, and do not interact with any other teams.

${ }^{4}$ The complete set of results are reported in the Appendix section.
} 
with communicative teams outperform those with diverse teams. As discussed earlier, PD systems need to have high integration capability (i.e. can manage integration costs) to attain best quality performance levels. Thus, for such projects, diverse teams tend to achieve superior best quality solutions than those communicative teams.

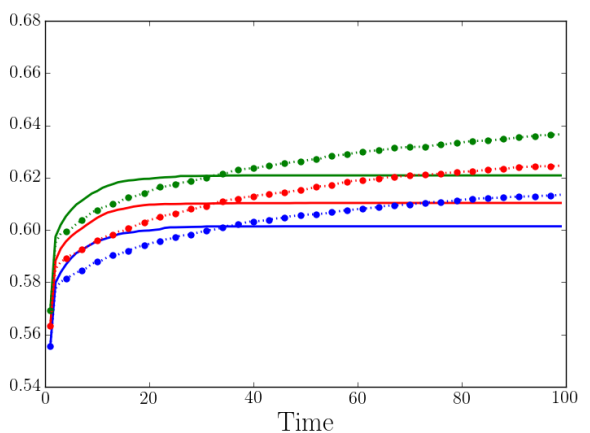

(a) $K=1, C=1$

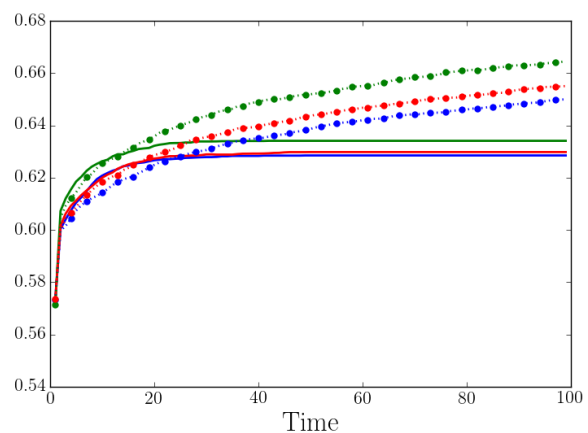

(b) $K=3, C=3$

Figure 2 The best quality performance of PD projects with communicative teams ( $\longrightarrow$ ) and those with diverse teams (-.-.-.-.-.-.), with subsystems interacting in modular (blue), cyclical (green), and acyclical (red). Each point is average performance of 100 simulation runs. In this figure, $n_{s}=n_{q}=5$ and $n_{e}=4$.

Graphs in Figure 3 depict the average quality performance $\left(V^{t}\right.$ in equation 5$)$ of PD projects with $K=C$ setting and the complete set of results are reported in the Appendix section. Plots indicate that when firms can attain average quality performance, two team-formation strategies result in different design performances. For all PD settings (e.g. interaction pattern, $K, C$ ), communicative teams tend to have higher average quality solutions than those of diverse teams. Interestingly, when PD systems are lowly capable, and consequently, can only attain average quality design solutions, communicative teams perform better than diverse teams. These results are totally different than those for projects capable of converging toward best quality designs. Hence, our result indicate that superior team formation strategy changes with PD system's capability level.

To investigate the seen patterns in graphs, paired t-tests are conducted to statistically compare performances across projects. Because each project type (e.g. diverse team strategy and $K=C=1$ ) is simulated on the same landscape as other type (e.g. communicative team strategy and $K=C=1$ ), the observations in one sample (e.g., performance of a project with diverse teams) can be paired with observations in another sample (e.g., performance of a project with communicative teams). As project's performance oscillates over time, we used the average PD performance in the last 10 time periods as performance measure in our statistical tests. We have conducted two paired t-tests. The 


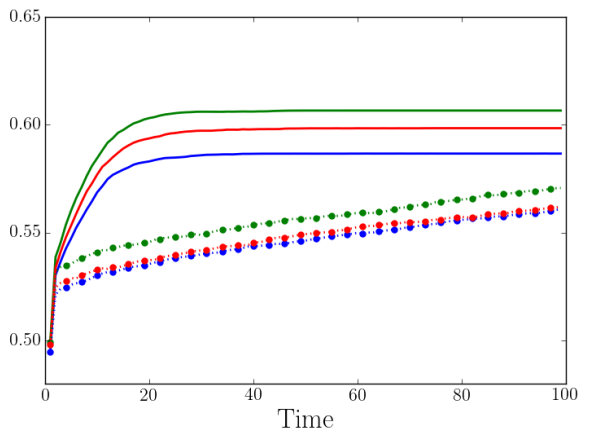

(a) $K=1, C=1$

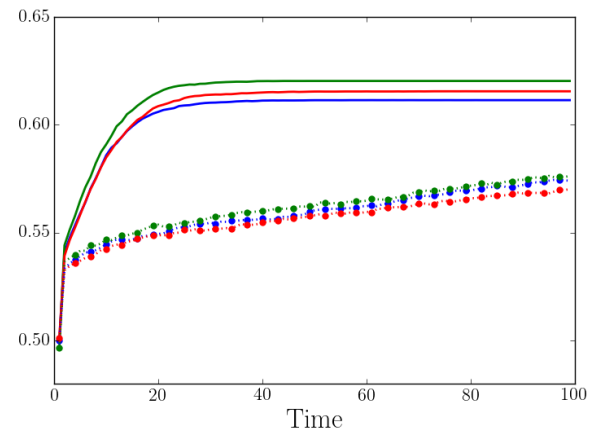

(b) $K=3, C=3$

Figure 3 The average quality performance of PD projects with communicative teams ( $\longrightarrow$ ) and those with diverse teams (-.---.-.---๑), with subsystems interacting in modular (blue), cyclical (green), and acyclical (red). Each point is average performance of $\mathbf{1 0 0}$ simulation runs. In this figure, $n_{s}=n_{q}=5$ and $n_{e}=4$.

null hypothesis for the first set of paired t-tests expects the mean of best quality of a system with diverse teams to be equal to that of a system with communicative teams ${ }^{5}$.

Th results of the first set of paired t-tests are provided in Table $2^{6}$. Clearly, the observed patterns in Figure 2 are echoed in the results. Especially, when comparing the bests design solutions found by communicative and diverse teams, those of the former teams tend to have lower fitness values than those of the latter teams. Across all PD settings (e.g. subsystems pattern, $K$ ), this result is robust and holds.

Table 2 Paired t-tests comparing the best quality performance of PD systems with communicative teams and those with diverse teams; For these PD systems, $n_{s}=n_{q}=5$ and $n_{e}=4$.

\begin{tabular}{|c|c|c|c|c|c|c|c|c|}
\hline \multicolumn{3}{|c|}{ Inputs } & \multirow{2}{*}{ Pattern } & \multirow{2}{*}{ t-value } & \multirow{2}{*}{ Pattern } & \multirow{2}{*}{ t-value } & \multirow{2}{*}{ Pattern } & \multirow{2}{*}{ t-value } \\
\hline$n_{q}$ & $K$ & $C$ & & & & & & \\
\hline \multirow{4}{*}{5} & 1 & 1 & \multirow{8}{*}{ Modular } & $-7.892^{*}$ & \multirow{8}{*}{ Cyclical } & $-9.125^{*}$ & \multirow{8}{*}{ Acyclical } & $-7.292^{*}$ \\
\hline & 1 & 2 & & $-11.698^{*}$ & & $-11.47^{*}$ & & $-8.157^{*}$ \\
\hline & 2 & 1 & & $-8.249^{*}$ & & $-12.305^{*}$ & & $-8.628^{*}$ \\
\hline & 2 & 2 & & $-10.094^{*}$ & & $-15.343^{*}$ & & $-11.565^{*}$ \\
\hline \multirow{4}{*}{7} & 1 & 1 & & $-5.118^{*}$ & & $-8.104^{*}$ & & $-6.555^{*}$ \\
\hline & 1 & 2 & & $-5.888^{*}$ & & $-10.517^{*}$ & & $-10.636^{*}$ \\
\hline & 2 & 1 & & $-7.564^{*}$ & & $-8.34^{*}$ & & $-8.939^{*}$ \\
\hline & 2 & 2 & & $-7.603^{*}$ & & $-10.246^{*}$ & & $-8.481^{*}$ \\
\hline
\end{tabular}

The null hypothesis of the second set of paired t-tests expects that there is no difference between the mean of average quality of a PD project with diverse teams and that of a project with communicative teams. These tests' results are shown in Table 3. Consistent with the graphs in Figure

${ }^{5}$ In other words, the first set of paired t-tests, compare $P Q^{t}$ in equation 4 of diverse teams with that of communicative teams. Therefore, positive and negative t-values indicate communicative teams are doing, respectively, better and worse than diverse teams.

${ }^{6}$ We use star $\left({ }^{*}\right)$ to indicate $1 \%$ significance level. 
3 , regarding the average design solutions found by communicative and diverse teams, those of the former have higher fitness values than those of the latter teams. This holds for all PD settings, e.g. subsystems pattern, $K, C$.

Table 3 Paired t-tests comparing the average quality performance of PD systems with communicative teams and those with diverse teams; For these PD systems, $n_{s}=n_{q}=5$ and $n_{e}=4$.

\begin{tabular}{|c|c|c|c|c|c|c|c|c|}
\hline \multicolumn{3}{|c|}{ Inputs } & \multirow{2}{*}{ Pattern } & \multirow{2}{*}{ t-value } & \multirow{2}{*}{ Pattern } & \multirow{2}{*}{ t-value } & \multirow{2}{*}{ Pattern } & \multirow{2}{*}{ t-value } \\
\hline$n_{q}$ & $K$ & $C$ & & & & & & \\
\hline \multirow{4}{*}{5} & 1 & 1 & \multirow{8}{*}{ Modular } & $14.724^{*}$ & \multirow{8}{*}{ Cyclical } & $18.423^{*}$ & \multirow{8}{*}{ Acyclical } & $16.213^{*}$ \\
\hline & 1 & 2 & & $14.044^{*}$ & & $19.551^{*}$ & & $18.781^{*}$ \\
\hline & 2 & 1 & & $16.11^{*}$ & & $20.671^{*}$ & & $14.725^{*}$ \\
\hline & 2 & 2 & & $16.027^{*}$ & & $20.882^{*}$ & & $23.895^{*}$ \\
\hline \multirow{4}{*}{7} & 1 & 1 & & $21.176^{*}$ & & $28.842^{*}$ & & $29.685^{*}$ \\
\hline & 1 & 2 & & $27.541^{*}$ & & $34.242^{*}$ & & $33.736^{*}$ \\
\hline & 2 & 1 & & $31.487^{*}$ & & $32.575^{*}$ & & $33.237^{*}$ \\
\hline & 2 & 2 & & $31.508^{*}$ & & $38.892^{*}$ & & $33.651^{*}$ \\
\hline
\end{tabular}

Our experimental results imply that depending on the integration capability and costs, PD managers should use either of the diverse and communicative teams. If PD systems can efficiently integrate different design solutions (e.g. low integration cost), diverse teams with high absorptive capacity should be assembled. However, for organizations with inefficient integration processes, this team formation strategy may not be superior. In the cases that large amount of resources (e.g. costs) are necessary to integrate different design solutions, managers should build communicative teams that, according to the average quality measure, tend to find design solutions with higher fitness values than those of the communicative teams.

It worths noting why the two team formation strategies make different performance trajectories/patterns. With deploying communicative teams, as designers are very likely to have similar expertise, the PD project confines its collective search effort to only parts of the whole landscape. As an example, consider an extreme case, when all designers in each team have the same expertise, or mathematically, $E X_{q}^{j}=E X^{j} \quad \forall d_{q}^{j} \in D_{j}$, and, there are five designers $\left(n_{q}=5\right)$ in each of the five $\left(n_{s}=5\right)$ teams, developing subsystems with $n_{e}=4$ elements. Then, such collective search effort, and in its full reach, eventually, can examine only $25 \times 2^{5}=800$ design solutions that is $\frac{800}{2^{20}}=0.076 \%$ of whole landscape.

Despite its confined search, using communicative teams has its own advantages. This strategy forms teams with high absorptive capacity, and their communications are highly effective. Thus, when a designer finds a superior design solution, that solution, quickly, and to an high extent, is communicated to the other designers.

With an imperfect imitation of design solutions, a number of designers converge and get close to the set of designers with superior designs (e.g. local optima). That number of designers that 
converge toward superior solutions (i.e. with high fitness values), by partially imitating their design solutions, is likely to be high at the initial stages of PD process since the designers' solutions are more likely to be inferior (i.e. the solutions are randomly selected). However, at the later steps of PD process, since designers' solutions have improved through either local search or communication, they oscillate between solutions near superior ones. That oscillation happens as designers partially imitate solution of an superior designer at a time, and then, later at another time, they partially imitate design solution of another superior designer. Those latter set of design solutions near a local peak is known as basin of attractions (Kauffman 1993, Levinthal 1997).

By building diverse teams, as designers are very likely to have different expertise, the PD project benefits from broaden collective search effort that covers mosts of the whole landscape. Consider an extreme case, when all designers in each team have the completely diverse expertise, or mathematically, $E X_{q}^{j} \cap E X_{q^{\prime}}^{j}=\emptyset \quad \forall d_{q}^{j}, d_{q^{\prime}}^{j} \in D_{j}$. Then, such collective search effort literally can examine whole landscape. Nevertheless, such search effort is limited by factors such as available resources (e.g. simulation time).

Despite its wide collective search, usage of diverse teams has its own drawbacks. This strategy forms teams with low absorptive capacity, and their communications are highly ineffective. Thus, although a designer may find a superior design solution, that design, slowly and ineffectively is communicated to the other designers. Hence, designers are less likely to benefit from the other designers' search effort.

The above arguments are confirmed by the following graphs in Figure $4^{7}$. Panels (a) and (b) show number of design elements communicated between designers at each time. Interestingly, the number of design elements communicated between designers in communicative teams increases at first and then decreases sharply. Conversely, number of communicated design elements for diverse teams show stable pattern, indicating that designers tendency to communicate does not change throughout PD process.

Additionally, we have calculated "within-team dissimilarity" of designers' solutions. Similar measures have been used in the literature (Fang et al. 2010, Schilling and Fang 2014), and we make pairwise comparisons of all $n_{q}$ designers in each team. Especially, each of $N$ design elements of those designers are compared using the following formula. In this equation, $\omega_{q 1, q 2, e}^{j}$ takes value 1 , if element $e$ of solutions of designers $d_{q 1}^{j}$ and $d_{q 2}^{j}$ are different, and value 0 , otherwise:

$$
D i s=\sum_{j=1}^{j=n_{s}} \sum_{q 1=1}^{q 1=n_{q}} \sum_{q 2=1}^{q 2=n_{q}} \sum_{e=1}^{e=n_{e}} \omega_{q 1, q 2, e}^{j} \forall d_{q 1}^{j}, d_{q 2}^{j} \in D_{j}
$$

\footnotetext{
7 These graphs illustrate results for PD projects with $K=C=1$ and $K=1, C=3$, and graphs for all PD settings are provided in the Appendix section. We observe similar pattern for all PD settings
} 
Panels (c) and (d) show within-team dissimilarity in equation 6 over time. According to these graphs, as designers find local optimum or superior solutions on the landscape, their solutions are imitated by the other designers, and consequently, within-team dissimilarity is decreasing for both communicative and diverse teams. By building communicative teams, within-team dissimilarity decreases quickly and, stabilizes at a very low level. Differently, however, within-team dissimilarity decreases at a lower rate when diverse teams are deployed.

Overall, the aggregated communicated elements and dissimilarity graphs in Figure 4, are consistent with our conjectures on the performance effects of using communicative and diverse teams. While communicative teams benefit from search effort of the other designers who have found superior solutions, diverse teams are more likely to find more superior solutions by having a broad and powerful collective search effort.

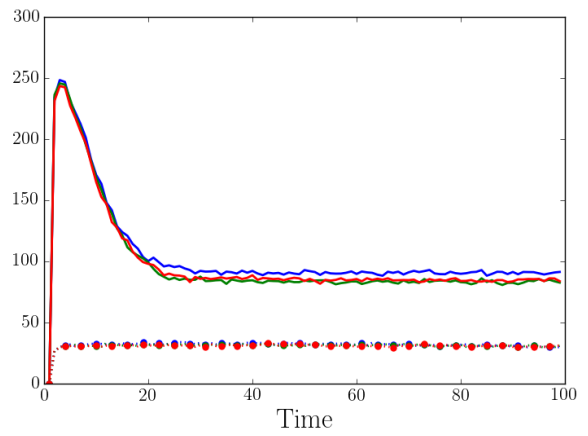

(a) $K=1, C=1$

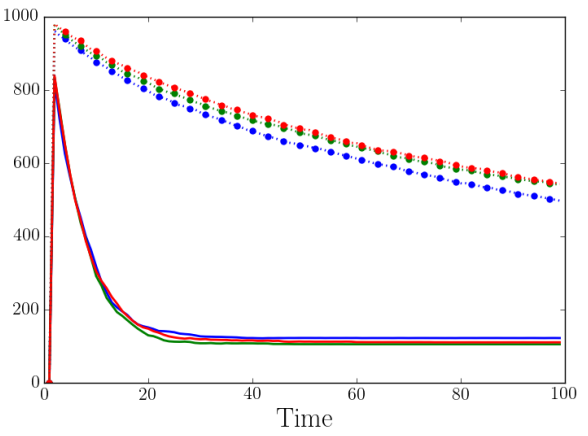

(c) $K=1, C=1$

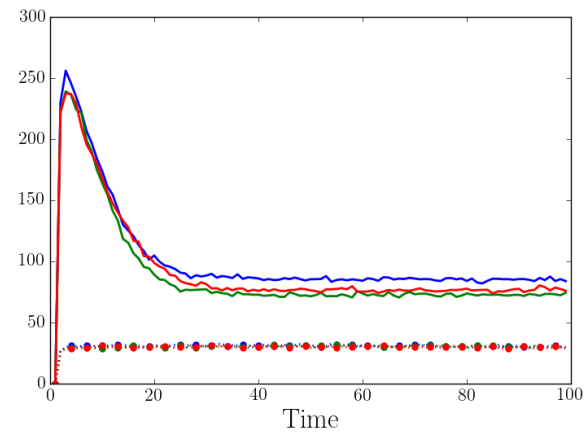

(b) $K=3, C=3$

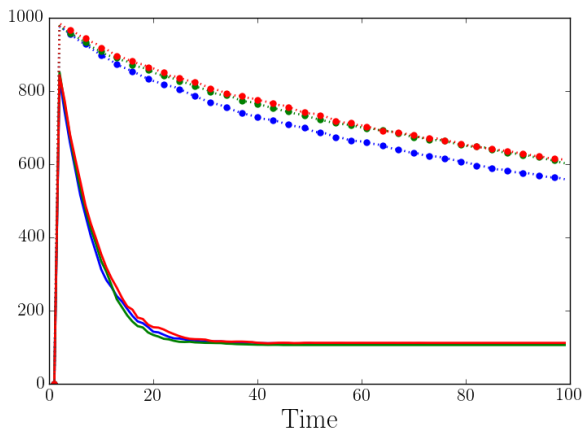

(d) $K=3, C=3$

Figure 4 The aggregated communicated elements (panels a and b) and within-team dissimilarity (panels c and d) of

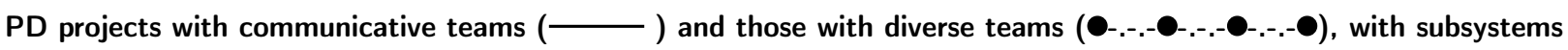
interacting in modular (blue), cyclical (green), and acyclical (red). Each point is average performance of 100 simulation runs. In this figure, $n_{s}=n_{q}=5$ and $n_{e}=4$. 
To identify micro-mechanisms that drive our results, we have conducted some other experiments. Recall that team formation strategies are characterized by considering two aspects of designers' search process: absorptive capacity level $\left(A C_{q^{\prime \prime} q^{\prime}}\right)$ and probability of designers having similar expertise domains $\left(P\left(E X_{q}^{j}=E X^{j}\right)\right)$. To that end, the corresponding values of these two features are fixed at a high value close to 1 , or, alternatively, a low value near 0 . Hence, these two features can be the main drivers of the previously seen performance behaviors. To clarify this argument, we have conducted two set of experiments, and their results are provided in the Appendix section.

In one set of experiments, we keep absorptive capacity level fixed $A C_{q^{\prime \prime} q^{\prime}}=A C \in[0,1]$, and then, redefine the two team formation strategies: (i) in communicative teams, or closely connected teams are deployed only by assuming a high probability for having similar expertise domains for all designers, and, (ii) in diverse teams or sparsely structured teams are assembled only by considering a low probability for designers having similar expertise domains. Next, we simulate PD systems with these two strategies for team assembly. The results are provided in the Appendix section.

In a similar experiments with different setups, we keep probability by which designers have similar expertise domains fixed $P\left(E X_{q}^{j}=E X^{j}\right)=P \in[0,1]$, and then, redefine the two team formation strategies: (i) in communicative teams or closely connected teams are deployed only by assuming a high absorptive capacity level for designers, and, (ii) in diverse teams or sparsely structured teams are assembled only with consideration of a low absorptive capacity level for designers. Next, we simulate PD systems with these two strategies for team assembly. The results are provided in the Appendix section.

Our results show that the best quality performance is concave in the absorptive capacity level $\left(A C_{q^{\prime \prime} q^{\prime}}=A C \in[0,1]\right)$, and also, is constant function of probability by which designers have similar expertise $\left(P\left(E X_{q}^{j}=E X^{j}\right)=P \in[0,1]\right)$. Similar performance patterns are seen for the average quality performance (see graphs in the Appendix section). Thus, absorptive capacity level appears to be highly critical factor in affecting PD performance than distribution of expertise among designers.

\subsection{Experiment 2: Teams with Heterogeneous Structures}

Managers may use the teams with heterogeneous structures, e.g. some teams with more closely connected structures develop some subsystems, whereas the other subsystems are developed by more diverse teams. In our second set of experiments, we investigate whether teams with heterogeneous structures - which we call them heterogeneous teams - result in higher PD performance than that of closely connected/diverse teams. Will PD projects have superior performance if they use teams with heterogeneous structures (i.e. having both diverse and communicative teams)? Does the answer to this question depend on either the interaction pattern among product subsystems or the level of interactions intensity? 
We compare the performance of a PD project where diverse teams developing core/peripheral with that of the former strategies (i.e. communicative teams or diverse teams). More precisely, we compare the resulting performance of teams with heterogeneous forms (i.e. diverse teams developing core/peripheral) with that of teams with uniform structures (i.e. communicative/diverse teams). We compare these strategies based on both the best and average quality measures that are defined in equation 4 and 5 . We simulate each scenario for a fixed number of time periods and record the performance measures of all teams.

As in our second set of experiments, we investigate strategies with heterogeneous forms according to their subsystems' interaction intensity, we limit the experiments with only cyclic and acyclic interaction patterns. In other words, as with modular pattern, to a large extent, teams have similar interaction intensity, we drop this pattern in our second set of experiments. Also, for definition of interaction strength measure, we use $W=5$ for the weight parameter in equation $2^{8}$. With this assumption, and under subsystems cyclic pattern and $n_{s}=5$, teams interaction intensity are as follows: $m_{1}=0.8, m_{2}=0.8, m_{3}=0.6, m_{4}=0.4, m_{5}=0.2$. However, subsystems acyclic pattern and $n_{s}=5$, teams interaction intensity are as: $m_{1}=0.66, m_{2}=0.53, m_{3}=0.4, m_{4}=0.26, m_{5}=0.13$.

For each combination (PD setting and subsystems' interaction pattern), we generate 100 landscapes. For each landscape, we create the corresponding PD project scenario (e.g. diverse teams developing core) and let teams conduct the search process for 100 time periods. This process is repeated for each of the 100 landscapes. The performance at each simulation time is the average performance at that time period across all landscapes.

Graphs in Figure 5 depict the best quality performance of PD projects ( $P Q^{t}$ in equation 4$)$. They show performance of PD projects with $K=C=1$. According to panels (a)-(b), heterogeneous teams (i.e. diverse teams developing core/peripheral) result in performance levels higher than or similar to that of diverse teams. In addition, panels (c)-(d) indicate that the former set of teams, i.e. with varied forms, tend to achieve higher performance levels than communicative teams. These observations are seen in the other PD projects (e.g. $K=3, C=1$ ) that are reported in the Appendix section. Thus, in $\mathrm{PD}$ systems with high integration capability that can attain best quality performance levels, heterogeneous teams are expected to achieve superior best quality solutions than uniformly formed teams, e.g. diverse/communicative teams.

Plots in Figure 6 indicate that when firms can attain average quality performance, heterogeneous teams have different performance courses, relative to that of uniformly formed teams, e.g. diverse/communicative teams. The performance graphs in panels (a)-(b) show that using heterogeneous teams results in performance levels higher than using diverse teams. In addition, panels

\footnotetext{
${ }^{8}$ The value of weight parameter only helps us to differentiate between teams under different interaction patterns.
} Thus, its value is likely to be trivial as long as teams can have differing interaction intensity levels. 


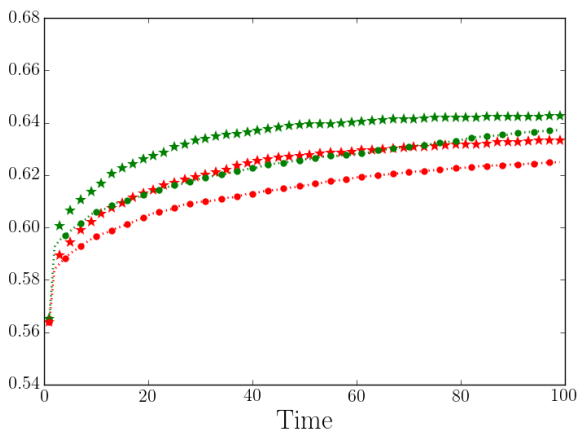

(a)

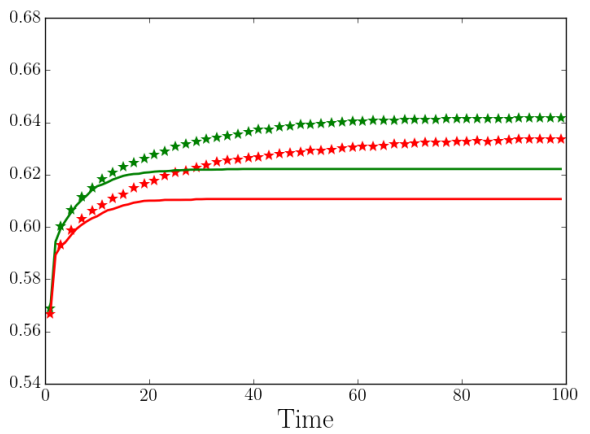

(c)

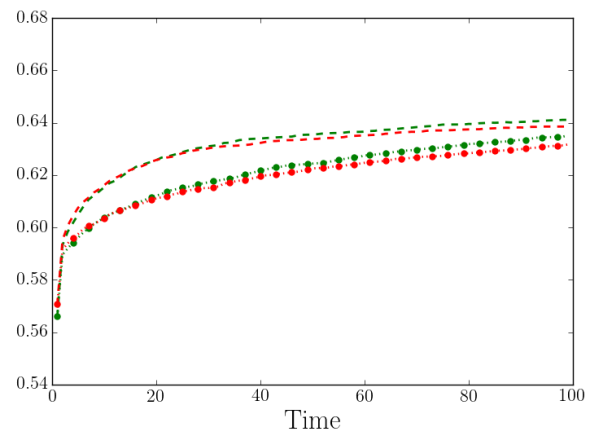

(b)

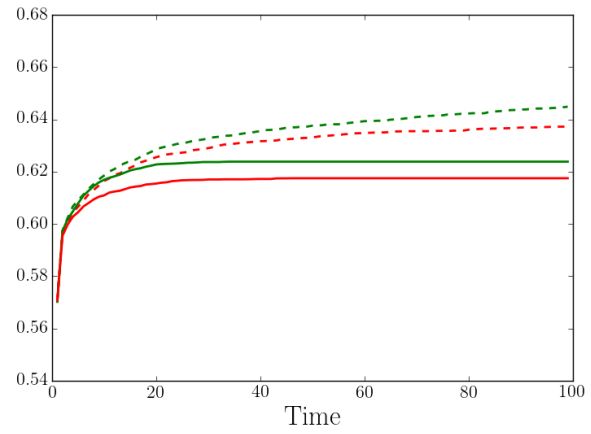

(d)

Figure 5 The best quality performance of PD projects with $K=1, C=1$, and: communicative teams (diverse teams ( -.-.--.--.--๑), diverse teams developing peripheral (*********) or core (- - - - - ). Subsystems interact in modular (blue), cyclical (green), and acyclical (red) patterns. Each point is average performance of 100 simulation runs, and $n_{s}=n_{q}=5, n_{e}=4$.

(c)-(d) indicate that, in short run, communicative teams achieve higher performance levels than that of heterogeneous teams. However, in the long run, the latter teams achieve higher performance than the former teams. These observations are seen in the other PD projects (e.g. $K=3, C=1$ ) that are reported in the Appendix section. Thus, in PD systems with low integration capability that can only achieve average quality performance levels, using heterogeneous teams, in the long run, PD systems are likely to make superior average quality solutions than deploying uniformly formed teams. However, in short term, communicative teams tend to attain higher average quality solutions than heterogeneous teams (e.g. diverse teams developing core).

To examine the previous performance patterns, we have conducted paired t-tests. Again, as discussed earlier in the previous section, the observations in one sample (e.g., performance of a project with teams whose diversity increases in interaction) can be paired with observations in another sample (e.g., performance of a project with communicative teams). As project's performance oscillates over time, we used the average PD performance in the last 10 time periods as performance 


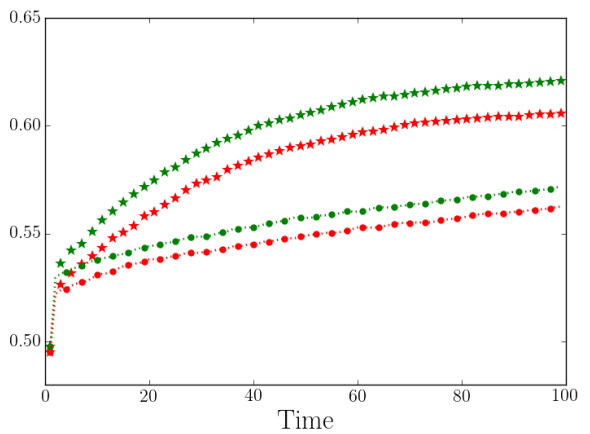

(a)

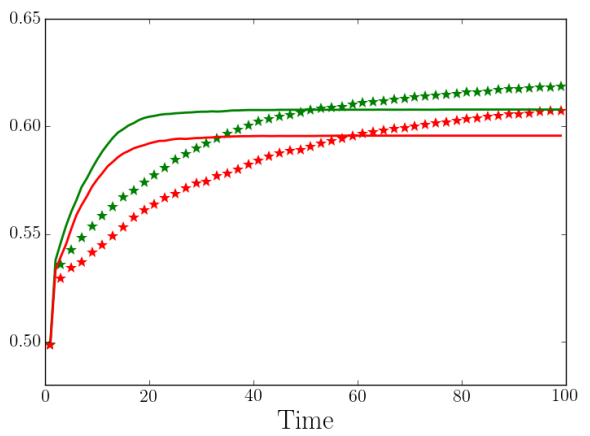

(c)

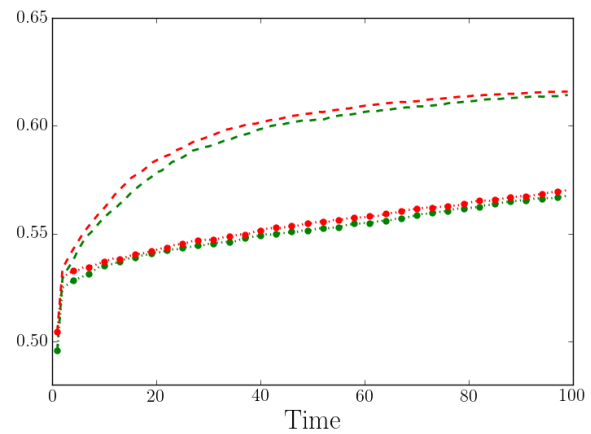

(b)

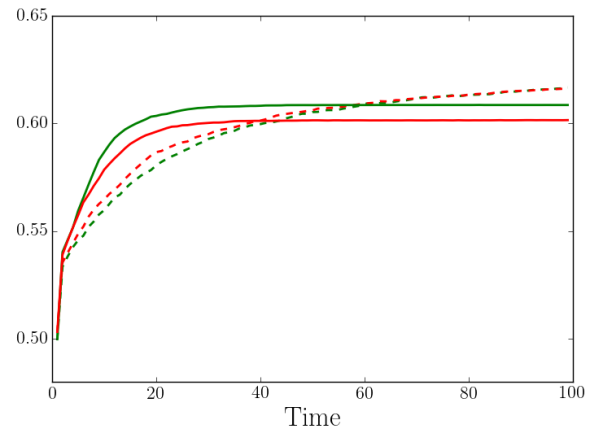

(d)

Figure 6 The average quality performance of PD projects with $K=1, C=1$, and: communicative teams (diverse teams ( -.-.--.--.--@), diverse teams developing peripheral (*********) or core (- - - - - ). Subsystems interact in modular (blue), cyclical (green), and acyclical (red) patterns. Each point is average performance of 100 simulation runs, and $n_{s}=n_{q}=5, n_{e}=4$.

measure in our statistical tests. We have conducted four set of paired t-tests. The null hypothesis for the first set of paired t-tests expects the mean of best quality of a system with diverse teams developing core/peripheral to be equal to that of a system with diverse teams ${ }^{9}$. Similarly, the second test expects the mean of best quality of the former system to be equal to that of a system with communicative teams.

Th results of the first and second paired t-tests are provided in Tables 4 and 5 for PD projects with $n_{q}=5$ designers in each team. The results show that heterogeneous teams (e.g. diverse teams developing core) achieve superior design solutions than that of communicative teams. However, in some PD settings, the best solutions of the former teams do not differ significantly from that

\footnotetext{
${ }^{9}$ In other words, the first set of paired t-tests, compare $P Q^{t}$ in equation 4 of diverse teams developing core with that of diverse teams. Therefore, positive and negative t-values indicate diverse teams developing core are doing, respectively, better and worse than diverse teams.
} 
of the diverse teams ${ }^{10}$. An example of such settings is the PD projects with acyclical subsystems interaction in some settings like $K=1, C=2$. These are consistent with the results in Section 4.1 where diverse teams are expected to find superior best solutions than communicative teams.

Table 4 Paired t-tests comparing the best quality performance of PD systems with diverse teams developing core and communicative/diverse teams; For these PD systems, $n_{s}=n_{q}=5$ and $n_{e}=4$.

\begin{tabular}{|c|c|c|c|c|c|c|}
\hline \multicolumn{3}{|l|}{ Inputs } & \multirow{2}{*}{ Pattern } & \multirow{2}{*}{ t-value } & \multirow{2}{*}{ Pattern } & \multirow{2}{*}{ t-value } \\
\hline Teams & $K$ & $C$ & & & & \\
\hline \multirow{4}{*}{ Diverse } & 1 & 1 & \multirow{8}{*}{ Cyclical } & $3.287^{*}$ & \multirow{8}{*}{ Acyclical } & $4.21^{*}$ \\
\hline & 1 & 2 & & -0.156 & & 1.662 \\
\hline & 2 & 1 & & 1.227 & & $2.803^{*}$ \\
\hline & 2 & 2 & & 0.034 & & 1.625 \\
\hline \multirow{4}{*}{ Communicative } & 1 & 1 & & $8.925^{*}$ & & $11.697^{*}$ \\
\hline & 1 & 2 & & $11.252^{*}$ & & $12.507^{*}$ \\
\hline & 2 & 1 & & $11.374^{*}$ & & $12.795^{*}$ \\
\hline & 2 & 2 & & $13.106^{*}$ & & $13.676^{*}$ \\
\hline
\end{tabular}

Table 5 Paired t-tests comparing the best quality performance of PD systems with diverse teams developing peripheral and communicative/diverse teams; For these PD systems, $n_{s}=n_{q}=5$ and $n_{e}=4$.

\begin{tabular}{|c|c|c|c|c|c|c|}
\hline \multicolumn{3}{|l|}{ Inputs } & \multirow{2}{*}{ Pattern } & \multirow{2}{*}{ t-value } & \multirow{2}{*}{ Pattern } & \multirow{2}{*}{ t-value } \\
\hline Teams & $K$ & $C$ & & & & \\
\hline \multirow{4}{*}{ Diverse } & 1 & 1 & \multirow{8}{*}{ Cyclical } & $2.968^{*}$ & \multirow{8}{*}{ Acyclical } & $5.085^{*}$ \\
\hline & 1 & 2 & & 1.62 & & 2.532 \\
\hline & 2 & 1 & & -0.034 & & $4.289^{*}$ \\
\hline & 2 & 2 & & 0.491 & & $3.873^{*}$ \\
\hline \multirow{4}{*}{ Communicative } & 1 & 1 & & $9.852^{*}$ & & $12.78^{*}$ \\
\hline & 1 & 2 & & $11.84^{*}$ & & $15.586^{*}$ \\
\hline & 2 & 1 & & $12.92^{*}$ & & $12.629^{*}$ \\
\hline & 2 & 2 & & $15.036^{*}$ & & $14.591^{*}$ \\
\hline
\end{tabular}

The null hypothesis for the third paired t-test expects the mean of average quality of systems with diverse teams developing core/peripheral to be equal to that of a system with diverse teams. Analogously, the fourth test expect the mean of average quality of the former systems to be equal to that of a system with communicative teams. For PD projects with $n_{q}=7$ designers in each team, these tests results are presented in Tables 6 and 7. They show that heterogeneous teams (e.g. diverse teams developing core) achieve higher average quality of solutions than that of diverse teams. However, in some PD settings, the best solutions of the former teams do not differ significantly from that of the communicative teams. An example of such settings is the PD projects with acyclical subsystems interaction in some settings like $K=1, C=2$.

\footnotetext{
${ }^{10}$ In the provided results in Appendix section, we observe somewhat different patterns for $n_{q}=7$ designers in each team, and we speculate that this happens as higher number of agents are conducting search over landscape, and intuitively, that may shift the overall performance improvement rate of heterogeneous teams in comparison to that of the uniformly formed teams. It worths noting, our overall insights are independent from these PD setting-related changes in results
} 
Table 6 Paired t-tests comparing the average quality performance of PD systems with diverse teams developing core and communicative/diverse teams; For these PD systems, $n_{s}=5, n_{q}=7$ and $n_{e}=4$.

\begin{tabular}{|c|c|c|c|c|c|c|}
\hline \multicolumn{3}{|l|}{ Inputs } & \multirow{2}{*}{ Pattern } & \multirow{2}{*}{ t-value } & \multirow{2}{*}{ Pattern } & \multirow{2}{*}{ t-value } \\
\hline Teams & $K$ & $C$ & & & & \\
\hline \multirow{4}{*}{ Diverse } & 1 & 1 & \multirow{8}{*}{ Cyclical } & $23.934^{*}$ & \multirow{8}{*}{ Acyclical } & $31.592^{*}$ \\
\hline & 1 & 2 & & $32.815^{*}$ & & $25.529^{*}$ \\
\hline & 2 & 1 & & $24.05^{*}$ & & $35.061^{*}$ \\
\hline & 2 & 2 & & $32.018^{*}$ & & $27.347^{*}$ \\
\hline \multirow{4}{*}{ Communicative } & 1 & 1 & & -0.826 & & 1.091 \\
\hline & 1 & 2 & & -2.296 & & -1.572 \\
\hline & 2 & 1 & & -1.09 & & 0.737 \\
\hline & 2 & 2 & & $-3.926^{*}$ & & -0.871 \\
\hline
\end{tabular}

Table 7 Paired t-tests comparing the average quality performance of PD systems with diverse teams developing peripheral and communicative/diverse teams; For these PD systems, $n_{s}=5, n_{q}=7$ and $n_{e}=4$.

\begin{tabular}{|c|c|c|c|c|c|c|}
\hline \multicolumn{3}{|l|}{ Inputs } & \multirow{2}{*}{ Pattern } & \multirow{2}{*}{ t-value } & \multirow{2}{*}{ Pattern } & \multirow{2}{*}{ t-value } \\
\hline Teams & $K$ & $C$ & & & & \\
\hline \multirow{4}{*}{ Diverse } & 1 & 1 & \multirow{8}{*}{ Cyclical } & $24.459^{*}$ & \multirow{8}{*}{ Acyclical } & $16.743^{*}$ \\
\hline & 1 & 2 & & $27.422^{*}$ & & $20.899^{*}$ \\
\hline & 2 & 1 & & $26.708^{*}$ & & $22.105^{*}$ \\
\hline & 2 & 2 & & $27.385^{*}$ & & $22.327^{*}$ \\
\hline \multirow{4}{*}{ Communicative } & 1 & 1 & & $-3.42^{*}$ & & $-5.452^{*}$ \\
\hline & 1 & 2 & & -1.87 & & $-7.148^{*}$ \\
\hline & 2 & 1 & & -2.057 & & $-6.42^{*}$ \\
\hline & 2 & 2 & & $-3.314^{*}$ & & $-6.838^{*}$ \\
\hline
\end{tabular}

Contemplating all four paired tests results, depending on organizational integration capability level, heterogeneous teams may achieve higher performance than teams with uniform structures. When PD systems have high competency in integrating designers solutions, then heterogeneous teams (e.g. diverse teams developing core) achieve superior design solutions than that of communicative teams. In addition, in PD projects have low integration capability, heterogeneous teams are expected to achieve higher performance than diverse teams. However, those heterogeneous teams may have higher or similar design solutions than that of diverse teams, in PD projects with high integration capability, and that of communicative teams in projects with low integration competency.

In sum, our model expected higher performance levels for projects with heterogeneous teams that utilize both diverse and communicative teams. Those PD projects benefits from superior features of both communicative and diverse teams. Recall that, with uniformly formed communicative teams, when a superior design solution is found, it is quickly and effectively shared among designers. Also, as discussed earlier, uniformly formed diverse teams utilize broad collective search effort. Hence, projects that employ heterogeneous teams have higher or, at least, similar, performance level to that of uniformly formed teams. 


\section{Discussion and Conclusion}

Organizations usually use teams as their means to conduct their product development projects. This paper proposes a model for investigating PD performance consequences of different team assembly strategies. Especially, according to the social network literature, two main teams structures are studied. In one structure, with a sparse structure, which we refer to as diverse teams, team members interact with designers who mainly do not interact with each other. Alternatively, with cohesive structure, or what we call communicative teams, designers interact in densely connected pattern where common-third parties are common.

Generally, in organization context, and particularly in the PD area, either of the two team assembly strategies is argued to be associated distinct performance advantages. For instance, communicative teams benefit from collaborative and high trust environment (Coleman 1990, Obstfeld 2005, Sosa 2011), whereas diverse teams provide designers diverse knowledge that are needed for generation of innovative ideas (Burt 2004, Fleming et al. 2007). Although, these general implications can guide PD managers, more research is needed to understand different PD team assembly strategies (Sosa and Marle 2013).

We investigate the team assembly strategies considering two important features of PD projects: product architecture and complexity level of its design space. Disentangling performance effects of product architecture or interaction pattern among subsystems has been a critical topic (MacCormack et al. 2006), and hence, we study performance effects of team assembly strategies with different product architecture patterns. To that end, we consider three different architectures, modular, cyclical and acyclical, that have been observed empirically (Baldwin and Clark 2000, MacCormack et al. 2006, Baldwin et al. 2014).

We also examine team formation policies for PD projects with different complexity levels as products can have design spaces with varying complexity levels or interdependencies among their elements (Fleming and Sorenson 2002). Generally, high NPD complexity creates challenges for managers that negatively impact performance such as profit margin (Tatikonda and Rosenthal 2000) and longer product development time (Griffin 1997). Theoretically, also, product complexity affects the relationship between the level of centralization of the product design decisions and product performance (Giannoccaro and Nair 2016). Therefore, in our model, we vary the level of ruggedness of design space to investigate whether team formation strategies affect PD performance in different ways.

We conceptualize PD endeavor as collective problem solving. That is designers are simultaneously solving a set of interrelated problems (Thomke 1997). More precisely, each designers conducts either of the following two tasks at a time: (i) locally optimizing his own design task, or (ii) attending design interactions with the other tasks (Mihm et al. 2003). Similar distributed problem-solving 
arrangements have been studied in organizational decision-making context (Lazer and Friedman 2007) where decision-makers interactions, rather than interactions of their tasks, are important. Following this line of work, we conceptualize design process as collective search over a performance landscape. More specifically, in our model, not only designers search activities are interdependent, but also, they interact but sharing their search attempt results.

From performance perspective, we study two performance measure to reflect different integration capability of firms. That differentiation between highly and lowly capable PD systems by using the best and average quality performance measures is in line with the fact that teams of knowledge workers need to integrate the members' knowledge in an ongoing process of mutual adjustment as their work progresses (Kozlowski et al. 1999, Gardner et al. 2012). The knowledge integration, in other words, makes some teams more effective than other teams by developing dynamic capabilities that enable the former teams to integrate knowledge in a systematic and reliable way (Gardner et al. 2012). Distinguishing the highly capable projects from the ones with low capability also seems plausible from the perspective of operational (e.g. adherence to schedule and budget) and product performance (e.g. financial and market performance ) measures (Blindenbach-Driessen et al. 2010, Tatikonda and Montoya-Weiss 2001). Highly capable projects can be those projects with high operational and product performances, and that occurs by identifying and integrating best design solutions. However, the low capable PD systems may be conceptualized as the ones with low operational and product performance levels, as they reach only to the average solutions.

It worths noting that our selection of two performance measures has no conflict with the fact that innovation success usually depends on the quality of the best identified opportunity (Girotra et al. 2010). More specifically, the best and average quality measures that is used in this paper, are built to differentiate PD projects based on their ability to discern the best design solutions which has been discussed in team and individual level (Girotra et al. 2010).

Table 8 provides a summary of our results on performance of PD teams that all of them are formed with a uniform strategy. Our results for projects where teams with similar social structures are utilized for all subsystems indicate that depending on the integration capability and costs, managers should use different strategies. If PD systems can efficiently integrate design solutions at low integration costs, diverse teams with high absorptive capacity should be assembled. However, with integration inefficiencies processes, managers should build communicative teams that, according to the average quality measure, tend to find design solutions with higher fitness values than those of the communicative teams.

Our results are in line with previous empirical observations and arguments. Previously, the differing consequences of spare networks with bridging ties and cohesive structures with strong ties are characterized as tension between "the idea problem" versus "the action problem" (Obstfeld 2005, 
Table 8 Comparing different team-formation strategies; “Heter” stands for Heterogeneous

\begin{tabular}{|c|c|c|}
\hline Team Formation & $\begin{array}{c}\text { Integration } \\
\text { Capability }\end{array}$ & $\begin{array}{c}\text { Superior Strategy } \\
\text { Uniform }\end{array}$ \\
High & $\begin{array}{c}\text { Diverse teams } \\
\text { Communicative teams }\end{array}$ \\
Leter-Uniform & High & Heter $>$ Communicative $(\geq$ Diverse $)$ \\
& Low & Heter $>$ Diverse $(\geq$ Communicative $)$ \\
\hline
\end{tabular}

Tiwana 2008). In other words, teams with cohesive structures, are capable of implementing innovative ideas, but are competent to generate ideas. Comparably, diverse teams with sparse structure have high capacity to generate innovative ideas but they are deficient in implementing ideas. Such trade-off has been seen in our results where communicative teams lost the potential to find the best quality solutions, and diverse teams missed the potential to have high average quality performance. In a similar perspective, and based on the creativity and decision-making literature, designers social network structure has been argued to affect the NPD front-end process (Khurana and Rosenthal 1998) in which ideas are born and further developed, ending with the go/no-go decision (Kijkuit and Van Den Ende 2007). In particular, diverse-team strategy is expected to improve idea generation, whereas communicative-team formation enhances idea development and evaluation processes.

Managers in PD projects may consider using teams with heterogeneous social structures. Our results in Table 8 provides a simple guideline on when such consideration is expected to result in higher PD performance than strategies that form teams uniformly. If PD systems can efficiently integrate design solutions at low integration costs, then heterogeneous teams attain superior best design solution than that of communicative teams. However, those variously formed teams are expected to have superior or similar best design solution as that of diverse teams. When integration processes are afflicted with inefficiencies, teams with heterogeneous social structures achieve higher average design solutions than that of diverse teams. Moreover, the former PD team arrangements attain higher than or similar to, the average design solutions of communicative teams.

In sum, our model expected higher performance levels for PD projects that employ from both diverse and communicative teams. This resonate with the body of research on association of social network features and innovation performance. For instance, Zhou et al. (2009) investigated a hightech firm in China, and found that too many or too few weak ties - that connect to different social circles and are source of non-redundant information (Granovetter 1973) - negatively affect creativity, and they suggested an inverted U-relationship between number of weak ties and creativity. Similarly, studies at individual level (Schultz and Schreyogg 2013), project level (Tiwana 2008), and firm level 
(Capaldo 2007) confirm that combination of weak ties (pervasive in our diverse teams) and strong ties (pervasive in our communicative teams) positively influence innovation performance.

Our results that expected higher performance of the heterogeneous teams, in comparison to that of uniformly formed teams, is also in line with arguments on small world fostering creativity (Fleming and Marx 2006). The small world structures benefit from both bridging ties and cohesive clusters that ease diffusion of new ideas, create more new inventions which will diffuse more quickly. Similarly, in the organizational context, groups with modest amounts of cross-group linking (i.e., low but nonzero levels) are found associated with higher equilibrium performance levels (Fang et al. 2010).

Our work has some implications for the literature on NK landscape search where managerial decision making problems (e.g. organization design, strategy planning) are conceptualized as problems for which the effective combinations of several interdependent variables should be found (Levinthal 1997, Katila and Ahuja 2002, Billinger et al. 2013). A stream of this research investigate how the search process features can have significant performance effects for problem solving in the complex settings (Fleming 2001, Nickerson and Zenger 2004). Expansion of search domain (Baumann and Siggelkow 2013), organizational structure (Knudsen and Levinthal 2007, Mihm et al. 2010), and interpersonal organization network (Lazer and Friedman 2007, Fang et al. 2010) are examples of such search process features. We add to this line of research by considering agents' communication effectiveness and expertise distribution in an interpersonal organization network. In particular, we have found that highly capable organizations should arrange their organizational structure using diverse teams who have high absorptive capacity. Conversely, organizations with high integration inefficiencies, should use communicative, and less diverse teams.

We conclude by discussing some limitations of our work and future research possibilities. First, NK landscape model impose some limitations on the number of teams and subsystems that we could include in our experiments, and feature research can validate our results by using alternative models like the recently developed NM landscape models (Buzas and Dinitz 2014, Manukyan et al. 2016) or organizational generalized pay-off functions (Fang et al. 2010, Schilling and Fang 2014) by which large landscape models, e.g. $N=50,100$ can be built.

Second, since a limited number of design elements could have been used in NK landscape, we used connected caveman model (Watts 1999) with a fixed structure. However, empirical research has shown that designers' interactions follow scale free properties - with a power of law degree distribution - and, those interactions are not equally distributed among all designers (Braha and Bar-Yam 2004). Hence, a few designers have a large number of interactions while the other ones have only few relationships. 
Lastly, we assume PD process is simultaneously executed for all subsystems. However, from temporal perspective, PD work might be front-loaded (Thomke and Fujimoto 2000) where problems are mainly solved in the early stage. Alternatively, the process can be back-loaded (Fixson and Marion 2012) with a large portion of problems being solved at the later stage of PD process. Investigating whether either of these temporal features affect the performance effects of team formation strategies can be a promising opportunity for future research.

\section{Acknowledgment}

This research was supported by Japan Society for the Promotion of Science (JSPS) grant.

\section{References}

Allen, T. J. 1977. Managing the flow of technology: Technology transfer and the dissemination of technological information within the R.ESD organization. MIT Press.

Ancona, D., and H. Bresman. 2013. X-teams: How to build teams that lead, innovate, and succeed. Harvard Business Press.

Anderson, E. G., and G. G. Parker. 2013. Integration of global knowledge networks. Production and Operations Management 22 (6): 1446-1463.

Baldwin, C., A. MacCormack, and J. Rusnak. 2014. Hidden structure: Using network methods to map system architecture. Research Policy 43 (8): 1381-1397.

Baldwin, C. Y., and K. B. Clark. 2000. Design rules: Volume 1, the power of modularity. The MIT Press.

Bardhan, I., V. V. Krishnan, and S. Lin. 2013. Team dispersion, information technology, and project performance. Production and Operations Management 22 (6): 1478-1493.

Barkoczi, D., M. Galesic, K. Katsikopoulos, P. Perrone, N. Ay, B. Straatman, B. Boyd, D. Mangalagiu, P. Rathje, C. Madsen et al. 2015. Social learning strategies reconcile the relationship between network structure and collective problem solving.

Baumann, O., and N. Siggelkow. 2013. Dealing with complexity: integrated vs. chunky search processes. Organization Science 24 (1): 116-132.

Billinger, S., N. Stieglitz, and T. R. Schumacher. 2013. Search on rugged landscapes: An experimental study. Organization Science 25 (1): 93-108.

Blindenbach-Driessen, F., J. Van Dalen, and J. Van Den Ende. 2010. Subjective performance assessment of innovation projects. Journal of Product Innovation Management 27 (4): 572-592.

Braha, D., and Y. Bar-Yam. 2004. Topology of large-scale engineering problem-solving networks. Physical Review E 69 (1): (016113)1-7.

Burt, R. S. 1992. Structural holes: The social structure of competition. MA: Harvard University Press.

Burt, R. S. 2004. Structural holes and good ideas1. American Journal of Sociology 110 (2): 349-399. 
Burt, R. S. 2005. Brokerage and closure: An introduction to social capital. Oxford University Press.

Buzas, J., and J. Dinitz. 2014. An analysis of landscapes: Interaction structure, statistical properties, and expected number of local optima. IEEE Transactions on Evolutionary Computation 18 (6): 807-818.

Capaldo, A. 2007. Network structure and innovation: The leveraging of a dual network as a distinctive relational capability. Strategic Management Journal 28 (6): 585-608.

Cohen, W. M., and D. A. Levinthal. 1990. Absorptive capacity: A new perspective on learning and innovation. Administrative Science Quarterly 35 (1): 128-152.

Coleman, J. 1990. Foundations of social theory.

Eppinger, S. D., D. E. Whitney, R. P. Smith, and D. A. Gebala. 1994. A model-based method for organizing tasks in product development. Research in Engineering Design 6 (1): 1-13.

Ethiraj, S. K., and D. Levinthal. 2004. Modularity and innovation in complex systems. Management Science 59 (2): 159-173.

Ethiraj, S. K., D. Levinthal, and R. R. Roy. 2008. The dual role of modularity: Innovation and imitation. Management Science 54 (5): 939-955.

Fang, C., J. Lee, and M. A. Schilling. 2010. Balancing exploration and exploitation through structural design: The isolation of subgroups and organizational learning. Organization Science 21 (3): 625-642.

Fixson, S. K., and T. J. Marion. 2012. Back-loading: A potential side effect of employing digital design tools in new product development. Journal of Product Innovation Management 29 (S1): 140-156.

Fleming, L. 2001. Recombinant uncertainty in technological search. Management science 47 (1): $117-132$.

Fleming, L., and M. Marx. 2006. Managing creativity in small worlds. California Management Review 48 (4): 6-27.

Fleming, L., S. Mingo, and D. Chen. 2007. Collaborative brokerage, generative creativity, and creative success. Administrative Science Quarterly 52 (3): 443-475.

Fleming, L., and O. Sorenson. 2002. Navigating the technology landscape of innovation. MIT Sloan Management Review 44 (2): 15-24.

Fujimoto, T. 1991. Product integrity and the role of designer-asintegrator. Design Management Journal (Former Series) 2 (2): 29-34.

Gardner, H. K., F. Gino, and B. R. Staats. 2012. Dynamically integrating knowledge in teams: Transforming resources into performance. Academy of Management Journal 55 (4): 998-1022.

Giannoccaro, I., and A. Nair. 2016. Examining the roles of product complexity and manager behavior on product design decisions: An agent-based study using nk simulation. IEEE Transactions on Engineering Management 63 (2): 237-247.

Gilbert, C. G. 2005. Unbundling the structure of inertia: Resource versus routine rigidity. Academy of Management Journal 48 (5): 741-763. 
Girotra, K., C. Terwiesch, and K. T. Ulrich. 2010. Idea generation and the quality of the best idea. Management Science 56 (4): 591-605.

Gokpinar, B., W. J. Hopp, and S. M. Iravani. 2013. In-house globalization: The role of globally distributed design and product architecture on product development performance. Production and Operations Management 22 (6): 1509-1523.

Granovetter, M. S. 1973. The strength of weak ties. American Journal of Sociology:1360-1380.

Griffin, A. 1997. The effect of project and process characteristics on product development cycle time. Journal of Marketing Research 34 (1): 24-35.

Griffin, A., and A. L. Page. 1996. PDMA success measurement project: recommended measures for product development success and failure. Journal of Product Innovation Management 13 (6): 478-496.

Griffith, T. L., J. E. Sawyer, and M. A. Neale. 2003. Virtualness and knowledge in teams: Managing the love triangle of organizations, individuals, and information technology. MIS Quarterly 27 (2): 265-287.

Guimera, R., B. Uzzi, J. Spiro, and L. A. N. Amaral. 2005. Team assembly mechanisms determine collaboration network structure and team performance. Science 308 (5722): 697-702.

Katila, R., and G. Ahuja. 2002. Something old, something new: A longitudinal study of search behavior and new product introduction. Academy of management journal 45 (6): 1183-1194.

Kauffman, S. A. 1993. The origins of order: Self organization and selection in evolution. Oxford University Press, USA.

Kavadias, S., and S. C. Sommer. 2009. The effects of problem structure and team diversity on brainstorming effectiveness. Management Science 55 (12): 1899-1913.

Khurana, A., and S. R. Rosenthal. 1998. Towards holistic "front end" in new product development. Journal of Product Innovation Management 15 (1): 57-74.

Kijkuit, B., and J. Van Den Ende. 2007. The organizational life of an idea: Integrating social network, creativity and decision-making perspectives. Journal of Management Studies 44 (6): 863-882.

Kilduff, M., and D. J. Brass. 2010. Organizational social network research: Core ideas and key debates. The Academy of Management Annals 4 (1): 317-357.

Knudsen, T., and D. A. Levinthal. 2007. Two faces of search: Alternative generation and alternative evaluation. Organization Science 18 (1): 39-54.

Knudsen, T., and K. Srikanth. 2014. Coordinated exploration organizing joint search by multiple specialists to overcome mutual confusion and joint myopia. Administrative Science Quarterly 59 (3): 409-441.

Kozlowski, S. W., S. M. Gully, E. R. Nason, and E. M. Smith. 1999. Developing adaptive teams: A theory of compilation and performance across levels and time. Pulakos (Eds.), The changing nature of performance: Implications for staffing, motivation, and development:240-292. 
Krishnan, V., and K. T. Ulrich. 2001. Product development decisions: A review of the literature. Management Science 47 (1): 1-21.

Lazer, D., and A. Friedman. 2007. The network structure of exploration and exploitation. Administrative Science Quarterly 52 (4): 667-694.

Levinthal, D. A. 1997. Adaptation on rugged landscapes. Management Science 43 (7): 934-950.

Liang, T.-P., J. Jiang, G. S. Klein, and J. Y.-C. Liu. 2010. Software quality as influenced by informational diversity, task conflict, and learning in project teams. IEEE Transactions on Engineering Management 57 (3): 477-487.

Lin, Y. 2014. Dynamics in organizational problem solving and the leveraging of social capital: An agent-based modeling (abm) perspective. Ph. D. thesis, University of Washington.

MacCormack, A., C. Baldwin, and J. Rusnak. 2012. Exploring the duality between product and organizational architectures: A test of the "mirroring" hypothesis. Research Policy 41 (8): 1309-1324.

MacCormack, A., J. Rusnak, and C. Y. Baldwin. 2006. Exploring the structure of complex software designs: An empirical study of open source and proprietary code. Management Science 52 (7): 1015-1030.

Manukyan, N., M. Eppstein, and J. Buzas. 2016. Tunably rugged landscapes with known maximum and minimum. IEEE Transactions on Evolutionary Computation 20 (2): 263 - 274.

Mihm, J., C. Loch, and A. Huchzermeier. 2003. Problem-solving oscillations in complex engineering projects. Management Science 46 (6): 733-750.

Mihm, J., C. Loch, D. Wilkinson, and B. A. Huberman. 2010. Hierarchical structure and search in complex organizations. Management Science 56 (5): 831-848.

Mishra, A., and K. K. Sinha. 2016. Work design and integration glitches in globally distributed technology projects. Production and Operations Management 25 (2): 347-369.

Nickerson, J. A., and T. R. Zenger. 2004. A knowledge-based theory of the firm-the problem-solving perspective. Organization Science 15 (6): 617-632.

Obstfeld, D. 2005. Social networks, the tertius iungens orientation, and involvement in innovation. Administrative Science Quarterly 50 (1): 100-130.

Parraguez, P., S. D. Eppinger, and A. M. Maier. 2015. Information flow through stages of complex engineering design projects: A dynamic network analysis approach. Engineering Management, IEEE Transactions on 62 (4): 604-617.

Quinn, J. B., and P. Pacquette. 1988. Ford: Team taurus. Teaching case. Dartmouth, NH: Amos Tuck School, Dartmouth College.

Reagans, R., and B. McEvily. 2003. Network structure and knowledge transfer: The effects of cohesion and range. Administrative Science Quarterly 48 (2): 240-267. 
Rivkin, J. W., and N. Siggelkow. 2007. Patterned interactions in complex systems: Implications for exploration. Management Science 53 (7): 1068-1085.

Sabbagh, K. 1996. Twenty first century jet: Making and marketing the boeing 7r7. Scribner Book Company.

Schilling, M. A., and C. Fang. 2014. When hubs forget, lie, and play favorites: Interpersonal network structure, information distortion, and organizational learning. Strategic Management Journal 35 (7): 974-994.

Schultz, C., and J. Schreyogg. 2013. The impact of network ties and resource input on research performance: An empirical investigation among surgeons in academic medical centers. IEEE Transactions on Engineering Management 60 (3): 457-468.

Sharman, D. M., and A. A. Yassine. 2004. Characterizing complex product architectures. Systems Engineering 7 (1): 35-60.

Simon, H. A. 1962. The architecture of complexity. Proceedings of the American Philosophical Society 106 (6): $467-482$.

Smith, R. P., and S. D. Eppinger. 1997. Identifying controlling features of engineering design iteration. Management Science 43 (3): 276-293.

Sosa, M. E. 2011. Where do creative interactions come from? the role of tie content and social networks. Organization Science 22:1-21.

Sosa, M. E. 2014. Realizing the need for rework: From task interdependence to social networks. Production and Operations Management 23 (8): 1312-1331.

Sosa, M. E., and F. Marle. 2013. Assembling creative teams in new product development using creative team familiarity. Journal of Mechanical Design 135 (8): (081009)1-13.

Sosa, M. E., J. Mihm, and T. R. Browning. 2013. Linking cyclicality and product quality. Manufacturing 83 Service Operations Management 15 (3): 473-491.

Tatikonda, M. V., and M. M. Montoya-Weiss. 2001. Integrating operations and marketing perspectives of product innovation: The influence of organizational process factors and capabilities on development performance. Management Science 47 (1): 151-172.

Tatikonda, M. V., and S. R. Rosenthal. 2000. Technology novelty, project complexity, and product development project execution success: a deeper look at task uncertainty in product innovation. IEEE Transactions on Engineering Management 47 (1): 74-87.

Thomke, S., and T. Fujimoto. 2000. The effect of front-loading problem-solving on product development performance. Journal of Product Innovation Management 17 (2): 128-142.

Thomke, S. H. 1997. The role of flexibility in the development of new products: An empirical study. Research policy 26 (1): 105-119.

Thompson, L. L. 2011. Making the team: A guide for managers. 
Tiwana, A. 2008. Do bridging ties complement strong ties? an empirical examination of alliance ambidexterity. Strategic Management Journal 29 (3): 251-272.

Tripsas, M., and G. Gavetti. 2000. Capabilities, cognition, and inertia: Evidence from digital imaging. Strategic Management Journal 21 (10-11): 1147-1161.

Tushman, M. L., and M. J. Peter. 1998. Dominant designs, technology cycles, and organization outcomes. In Academy of Management Proceedings, Volume 1998, A1-A33. Academy of Management.

Tushman, M. L., and L. Rosenkopf. 1992. Organizational determinants of technological-change-toward a sociology of technological evolution. Research in Organizational Behavior 14:311-347.

Ulrich, K. 1995. The role of product architecture in the manufacturing firm. Research policy 24 (3): 419-440.

Uzzi, B. 1997. Social structure and competition in interfirm networks: The paradox of embeddedness. Administrative Science Quarterly 42 (1): 35-67.

Von Hippel, E. 1990. Task partitioning: An innovation process variable. Research policy 19 (5): 407-418.

Vuori, T. O., and Q. N. Huy. 2015. Distributed attention and shared emotions in the innovation process how nokia lost the smartphone battle. Administrative Science Quarterly.

Watts, D. J. 1999. Networks, dynamics, and the small-world phenomenon 1. American Journal of sociology 105 (2): 493-527.

Weinberger, E. D. 1991. Local properties of Kauffman's NK model: A tunably rugged energy landscape. Physical Review A 44 (10): 6399.

Young-Hyman, T. 2016. Cooperating without co-laboring how formal organizational power moderates crossfunctional interaction in project teams. Administrative Science Quarterly.

Zhou, J., S. J. Shin, D. J. Brass, and J. Choi. 2009. Social networks, personal values, and creativity: Evidence for curvilinear and interaction effects. Journal of Applied Psychology 94 (6): 1544-1552. 


\section{Appendix}

Experiment 1: Figures

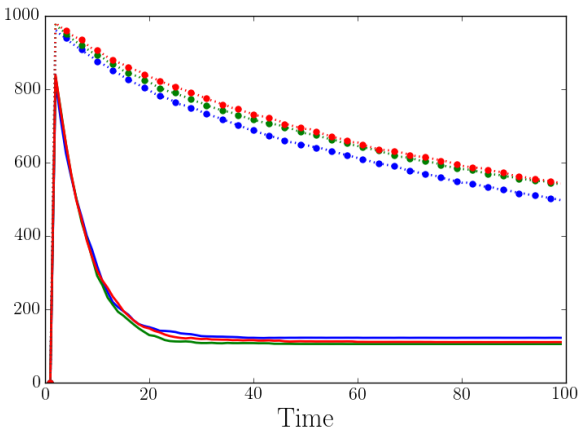

(a) $K=1, C=1$

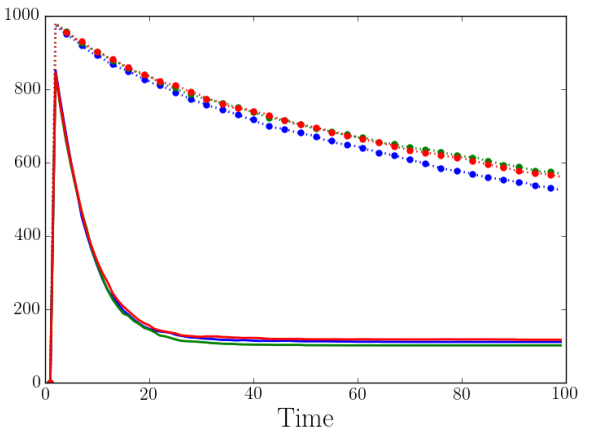

(c) $K=3, C=1$

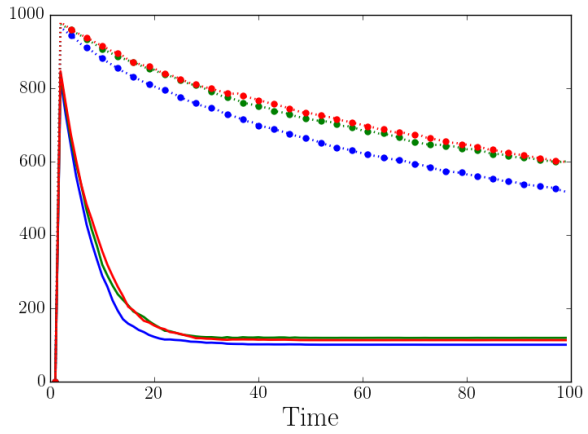

(b) $K=1, C=3$

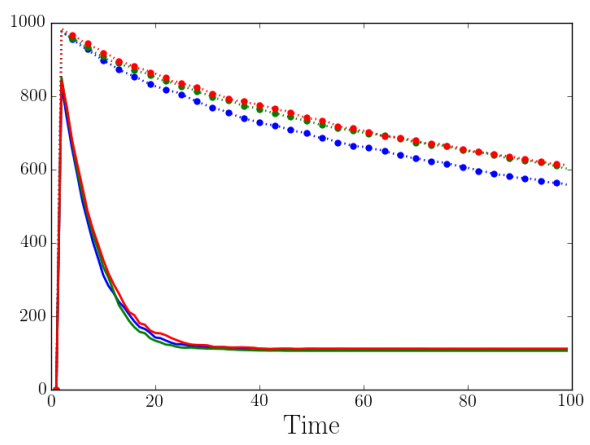

(d) $K=3, C=3$

Figure 7 The within-team dissimilarity of PD projects with communicative teams $(-)$ and those with diverse teams (-.-.-.-.-.-@), with subsystems interacting in modular (blue), cyclical (green), and acyclical (red). Each point is average performance of 100 simulation runs. In this figure, $n_{s}=n_{q}=5$ and $n_{e}=4$. 


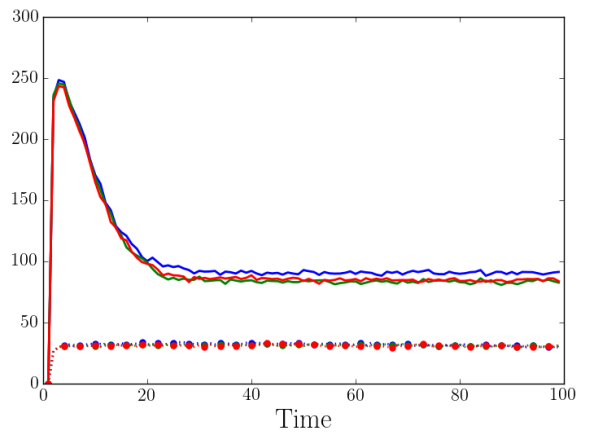

(a) $K=1, C=1$

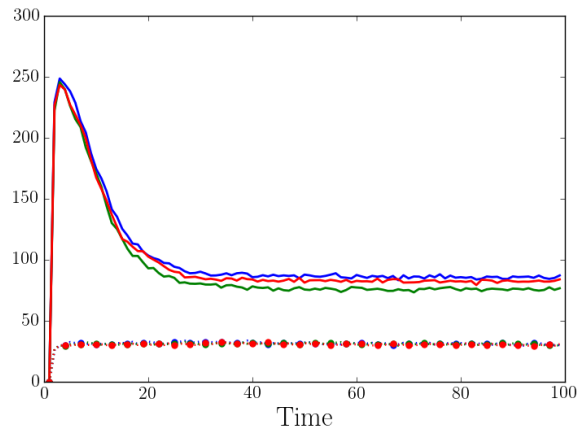

(c) $K=3, C=1$

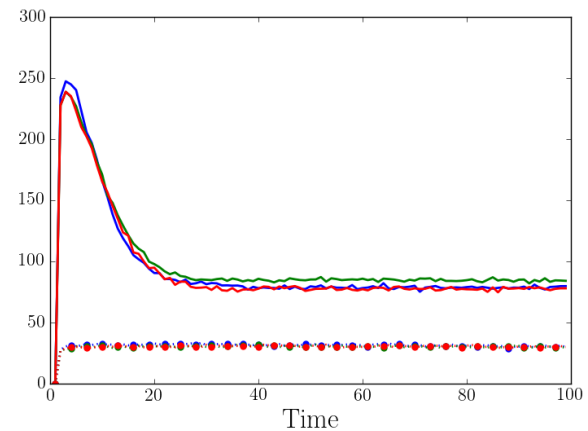

(b) $K=1, C=3$

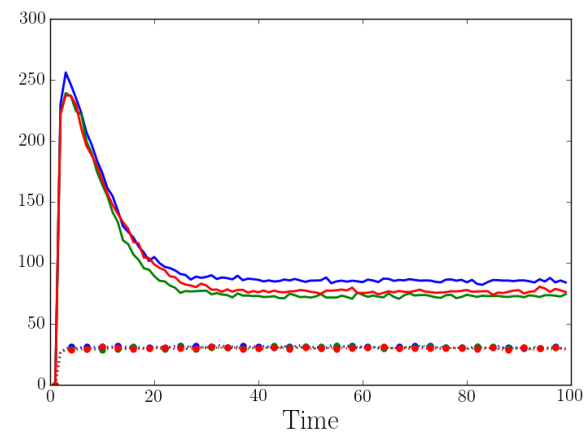

(d) $K=3, C=3$

Figure 8 The aggregated communicated elements of PD projects with communicative teams $\left(-\frac{}{-}\right)$ and those with diverse teams (-.-.-.-.-.--), with subsystems interacting in modular (blue), cyclical (green), and acyclical (red). Each point is average performance of $\mathbf{1 0 0}$ simulation runs. In this figure, $n_{s}=n_{q}=5$ and $n_{e}=4$. 


\section{Experiment1: Micro mechanisms}

To identify micro-mechanisms that drive our results, we have conducted some other experiments. Recall that team formation strategies are characterized by considering two aspects of designers' search process: absorptive capacity level $\left(A C_{q^{\prime \prime} q^{\prime}}\right)$ and probability of designers having similar expertise domains $\left(P\left(E X_{q}^{j}=E X^{j}\right)\right)$. To that end, the corresponding values of these two features are fixed at a high value close to 1 , or, alternatively, a low value near 0 . Hence, these two features can be the main drivers of the previously seen performance behaviors. To clarify this argument, we have conducted two set of experiments.

In one set of experiments, we keep absorptive capacity level fixed $A C_{q^{\prime \prime} q^{\prime}}=A C \in[0,1]$, and then, redefine the two team formation strategies: (i) in forming communicative teams, closely connected teams are deployed only by assuming a high probability for having similar expertise domains for all designers, and, (ii) in forming diverse teams, sparsely structured teams are assembled only by considering a low probability for designers having similar expertise domains. Next, we simulate PD systems with these two strategies for team assembly. The final performance of these projects according to the best quality measure, are shown in Figures 9 and 11. In these graphs, $\mathrm{x}$-axis shows absorptive capacity level that varies in the following range $A C \in[0.1,0.2,0.3, . ., 0.9]$. As shown in panels (a) and (b), the best quality performance is higher when the absorptive capacity level is low (i.e. $A C=0.1)$ than when it is high $(A C=0.9)$. This is consistent with the previous pattern where diverse teams achieve higher best quality performance than communicative teams.

In a similar experiments with different setups, we keep probability by which designers have similar expertise domains fixed $P\left(E X_{q}^{j}=E X^{j}\right)=P \in[0,1]$, and then, redefine the two team formation strategies: (i) in forming communicative teams, closely connected teams are deployed only by assuming a high absorptive capacity level for designers, and, (ii) in forming diverse teams, sparsely structured teams are assembled only with consideration of a low absorptive capacity level for designers. Next, we simulate PD systems with these two strategies for team assembly. The final performance of these projects according to the best quality measure, are shown in Figures 10 and 12. In these graphs, $\mathrm{x}$-axis shows probability by which designers have similar expertise, and it varies in the following range $P \in[0.1,0.2,0.3, . ., 0.9]$.

The graphs in Figures 9-12 show that the best quality performance is concave in the absorptive capacity level $\left(A C_{q^{\prime \prime} q^{\prime}}=A C \in[0,1]\right)$, and also, is constant function of probability by which designers have similar expertise $\left(P\left(E X_{q}^{j}=E X^{j}\right)=P \in[0,1]\right)$. Similar performance patterns are seen for the average quality performance (see graphs in the Appendix section). Thus, absorptive capacity level appears to be highly critical factor in affecting $\mathrm{PD}$ performance than distribution of expertise among designers. 


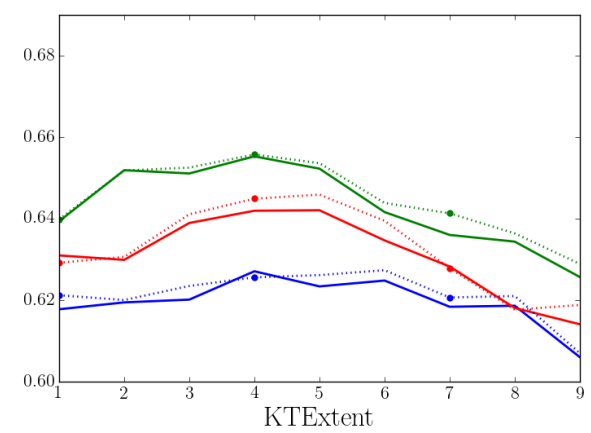

(a) $K=1, C=1$

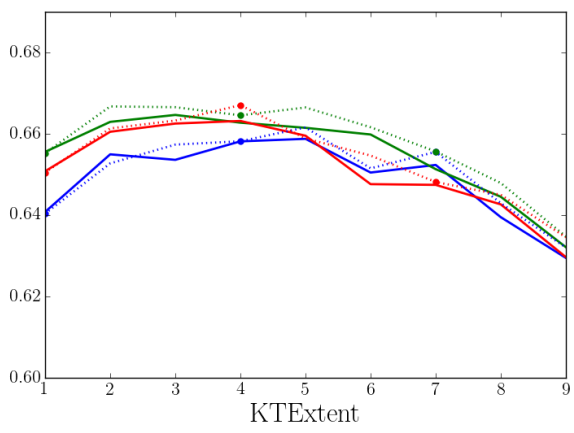

(c) $K=3, C=1$

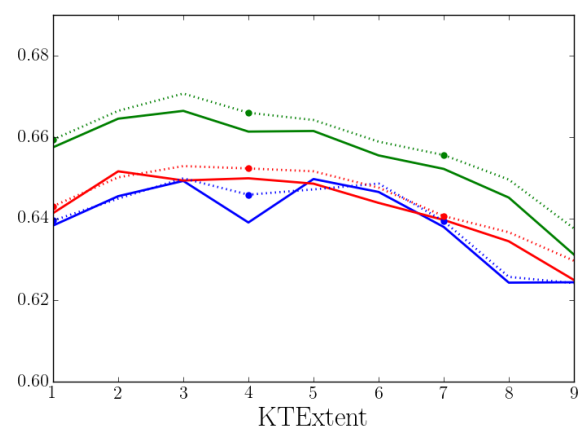

(b) $K=1, C=3$

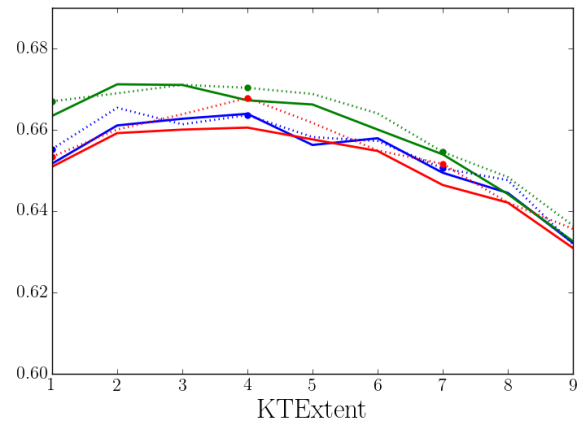

(d) $K=3, C=3$

Figure 9 The best quality performance of PD projects over fixed absorptive capacity $A C$. These graphs illustrate performance of the communicative teams (— ) and those with diverse teams (-..-.-..-.-..-@) with subsystems interacting in modular (blue), cyclical (green), and acyclical (red). Each point is average performance of 100 simulation runs. In this figure, $n_{s}=n_{q}=5$ and $n_{e}=4$. 


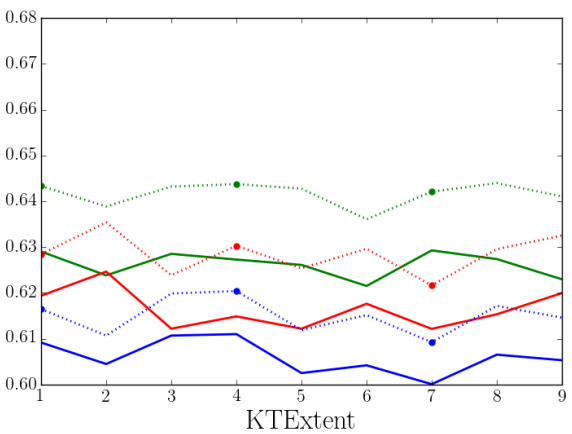

(a) $K=1, C=1$

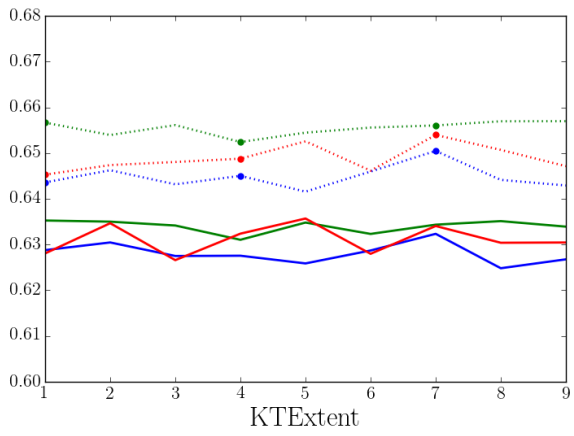

(c) $K=3, C=1$

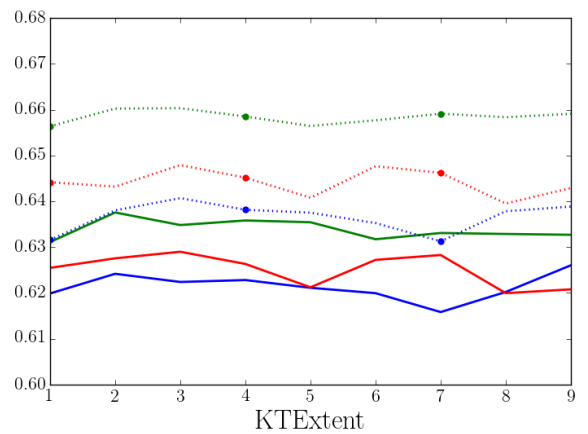

(b) $K=1, C=3$

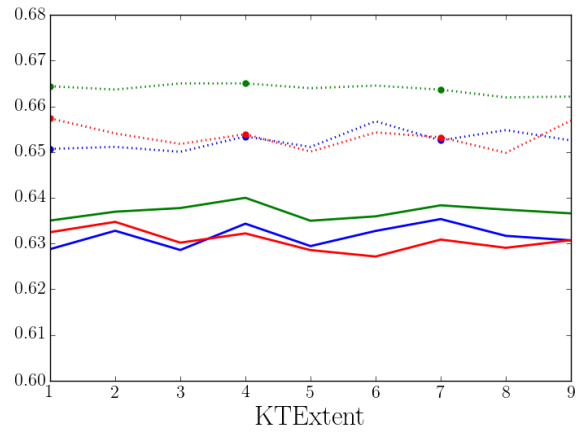

(d) $K=3, C=3$

Figure 10 The best quality performance of PD projects over expertise distribution probability. These graphs illustrate performance of the communicative teams (— ) and those with diverse teams (-..-.-.-.-.-.-@) with subsystems interacting in modular (blue), cyclical (green), and acyclical (red). Each point is average performance of 100 simulation runs. In this figure, $n_{s}=n_{q}=5$ and $n_{e}=4$. 


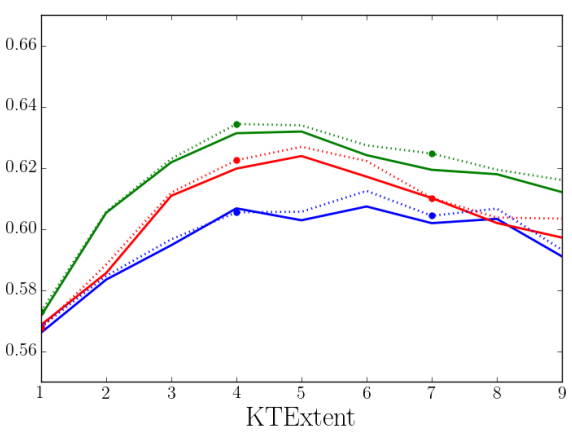

(a) $K=1, C=1$

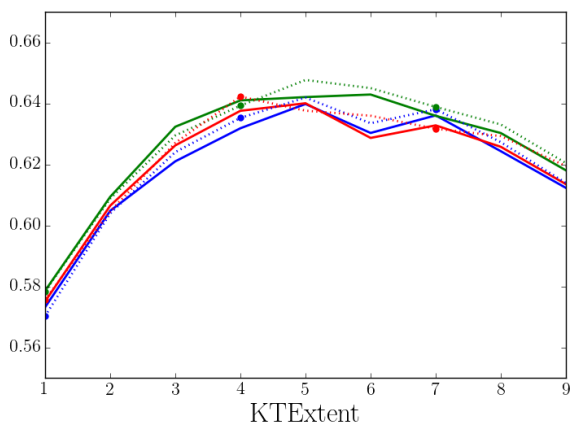

(c) $K=3, C=1$

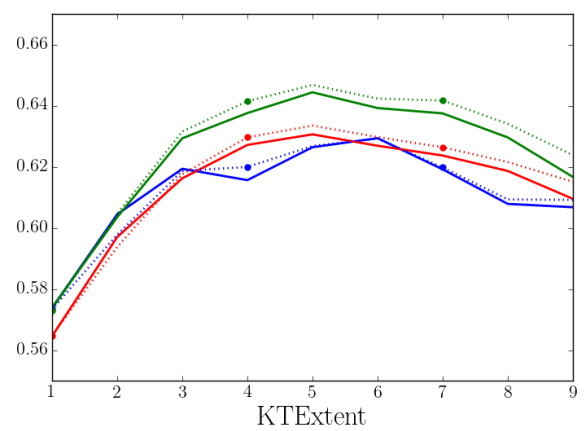

(b) $K=1, C=3$

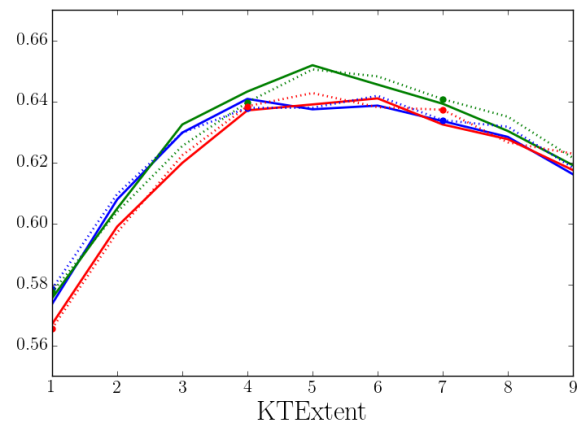

(d) $K=3, C=3$

Figure 11 The average quality performance of PD projects over fixed absorptive capacity $A C$. These graphs illustrate performance of the communicative teams ( ) and those with diverse teams (

-.-.--.-.-.-.-) with subsystems interacting in modular (blue), cyclical (green), and acyclical (red). Each point is average performance of 100 simulation runs. In this figure, $n_{s}=n_{q}=5$ and $n_{e}=4$. 


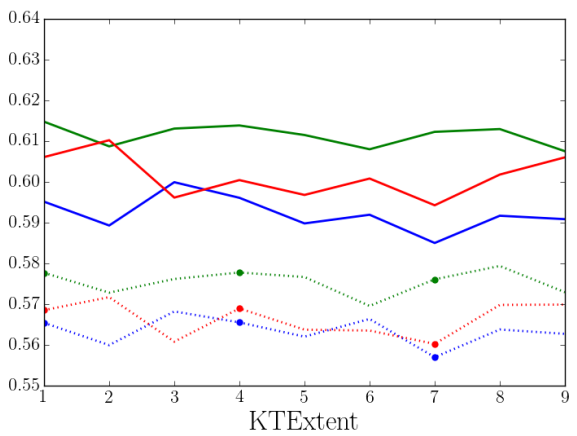

(a) $K=1, C=1$

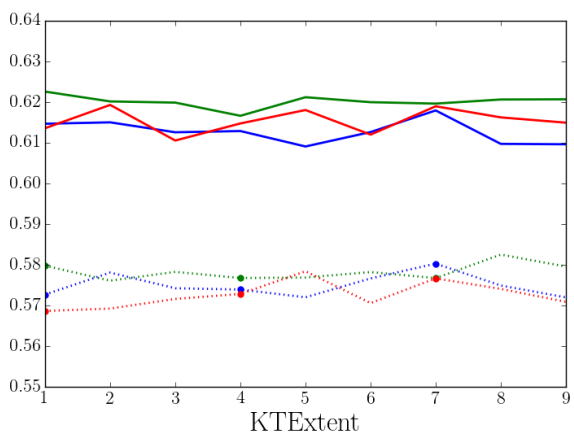

(c) $K=3, C=1$

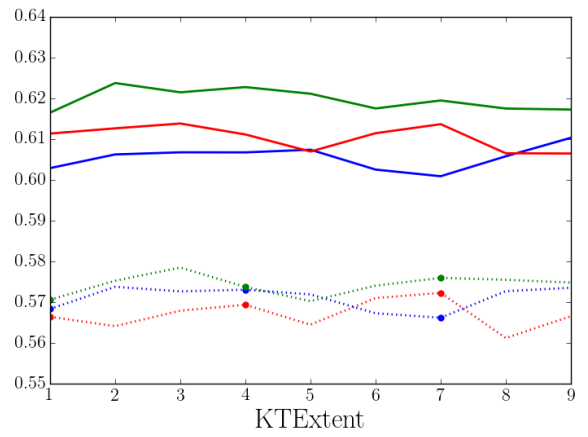

(b) $K=1, C=3$

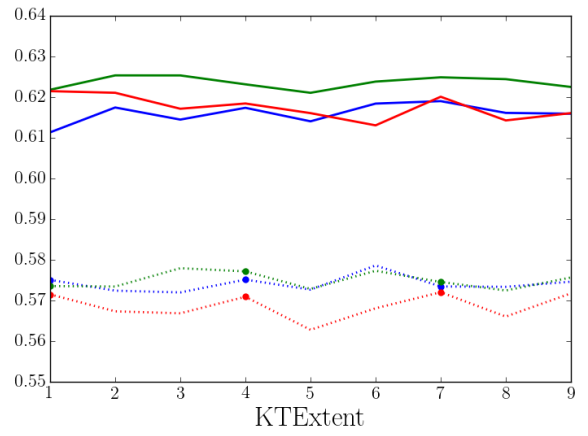

(d) $K=3, C=3$

Figure 12 The average quality performance of PD projects over expertise distribution probability. These graphs illustrate performance of the communicative teams ( ) and those with diverse teams ( subsystems interacting in modular (blue), cyclical (green), and acyclical (red). Each point is average performance of 100 simulation runs. In this figure, $n_{s}=n_{q}=5$ and $n_{e}=4$. 


\section{Experiment 2: Figures}

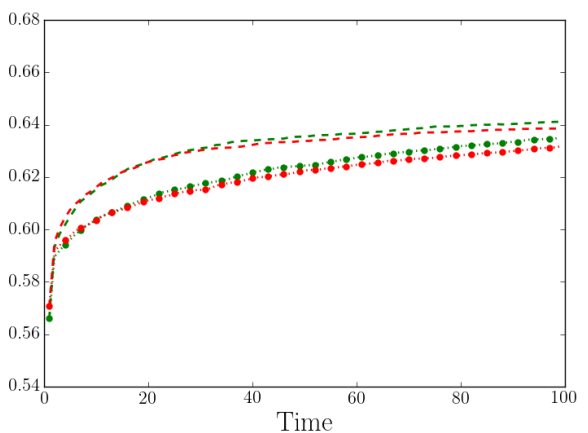

(a) $K=1, C=1$

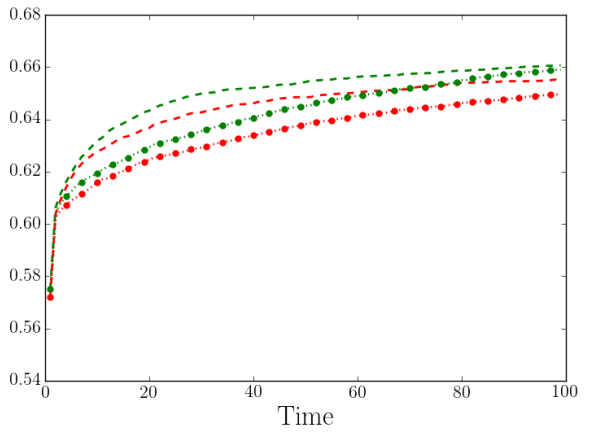

(c) $K=3, C=1$

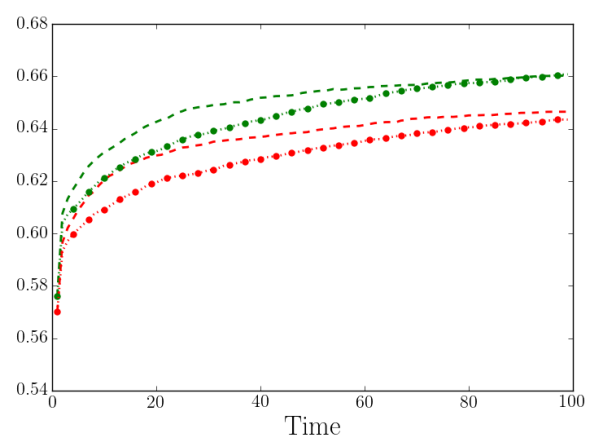

(b) $K=1, C=3$

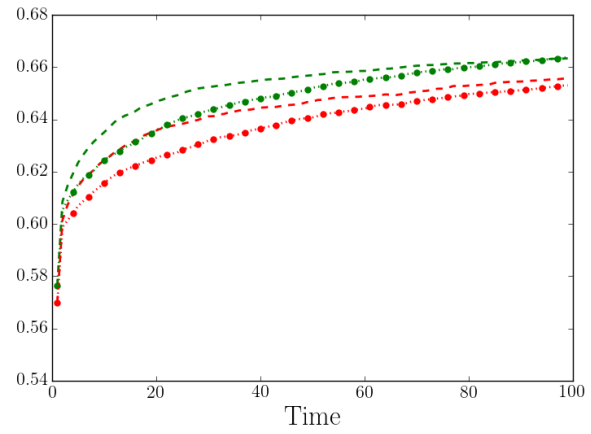

(d) $K=3, C=3$

Figure 13 The best quality performance of PD projects with diverse teams ( developing core (- - - - - ). Subsystems interact as modular (blue), cyclical (green), and acyclical (red) patterns.

Each point is average performance of 100 simulation runs, and $n_{s}=n_{q}=5, n_{e}=4$. 


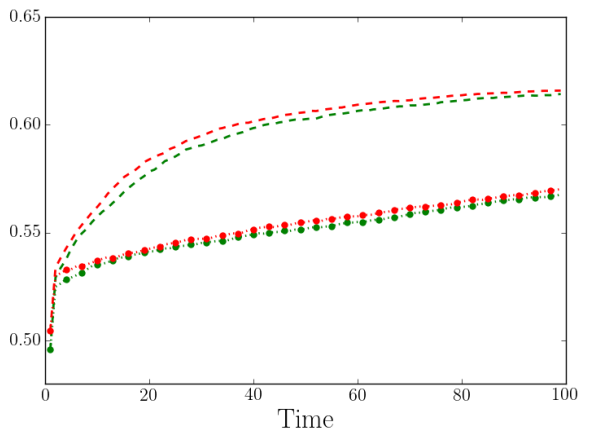

(a) $K=1, C=1$

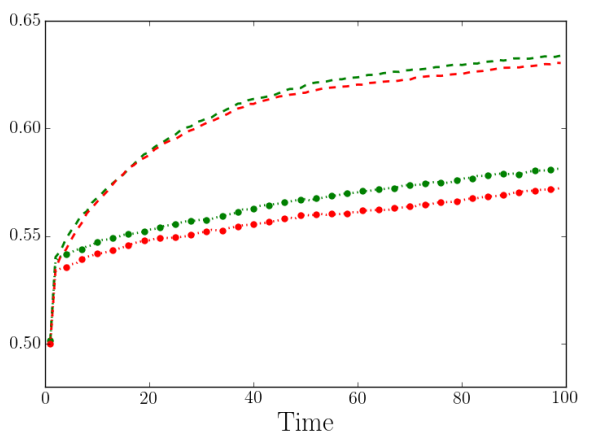

(c) $K=3, C=1$

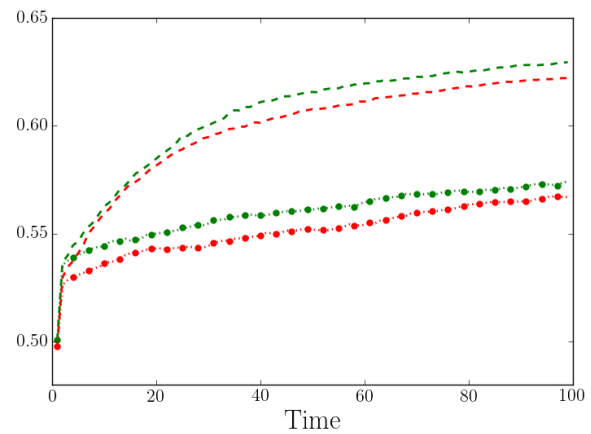

(b) $K=1, C=3$

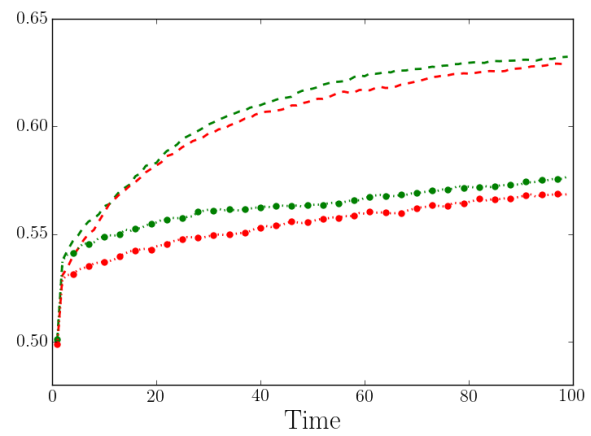

(d) $K=3, C=3$

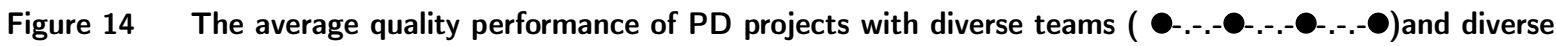
teams developing core (- - - - - -). Subsystems interact as modular (blue), cyclical (green), and acyclical (red) patterns. Each point is average performance of $\mathbf{1 0 0}$ simulation runs, and $n_{s}=n_{q}=5, n_{e}=4$. 


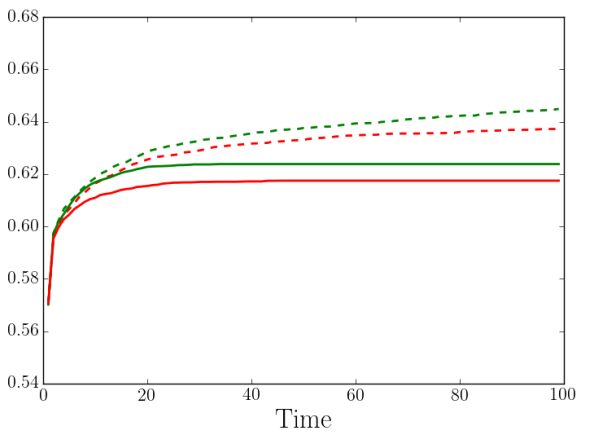

(a) $K=1, C=1$

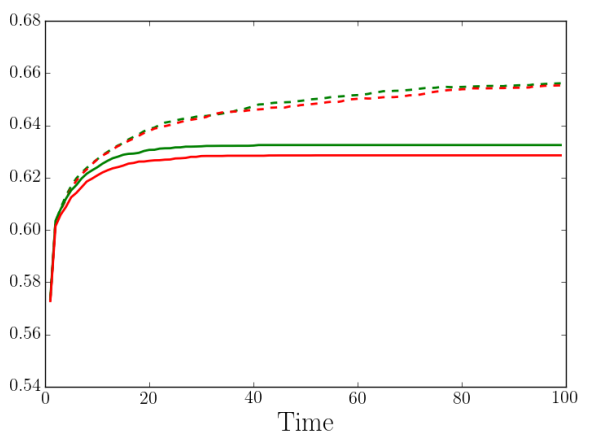

(c) $K=3, C=1$

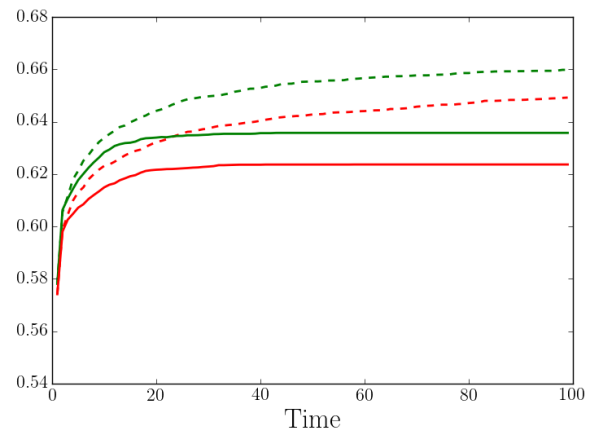

(b) $K=1, C=3$

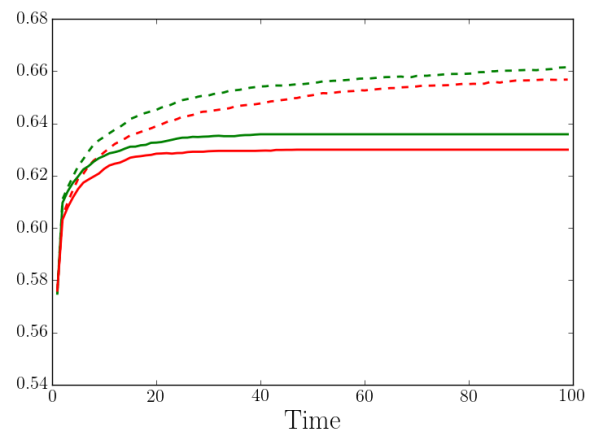

(d) $K=3, C=3$

Figure 15 The best quality performance of PD projects with communicative teams ( - ) and diverse teams developing core (- - - - - -). Subsystems interact as modular (blue), cyclical (green), and acyclical (red) patterns. Each point is average performance of 100 simulation runs, and $n_{s}=n_{q}=5, n_{e}=4$. 


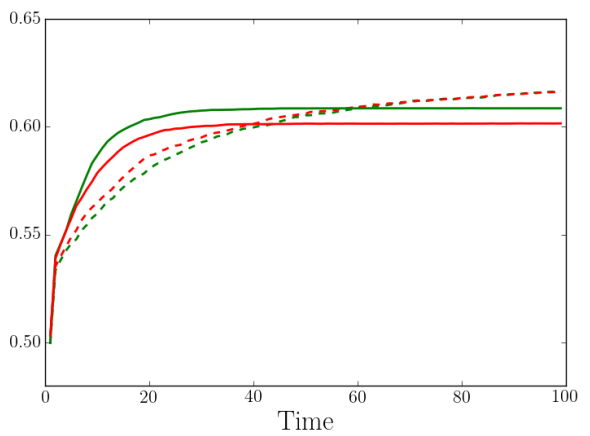

(a) $K=1, C=1$

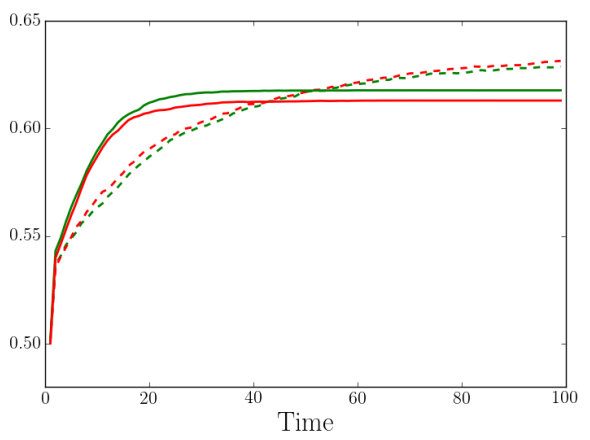

(c) $K=3, C=1$

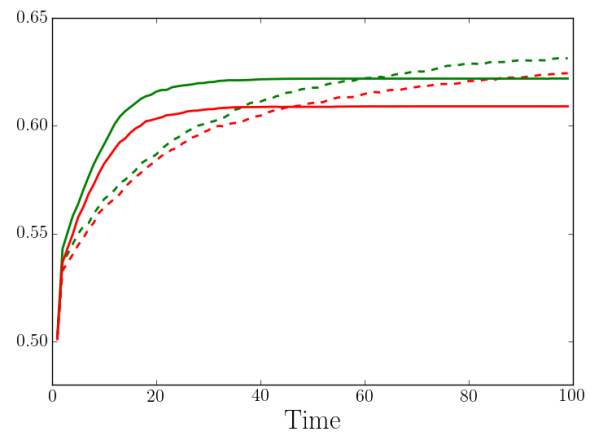

(b) $K=1, C=3$

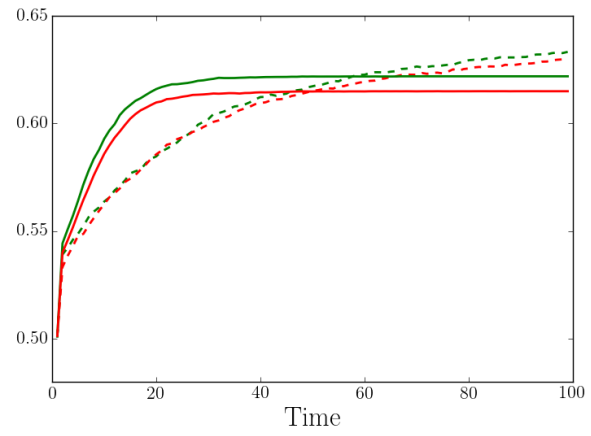

(d) $K=3, C=3$

Figure 16 The average quality performance of PD projects with communicative teams ( - ) and diverse teams developing core (- - - - - -). Subsystems interact as modular (blue), cyclical (green), and acyclical (red) patterns.

Each point is average performance of 100 simulation runs, and $n_{s}=n_{q}=5, n_{e}=4$. 


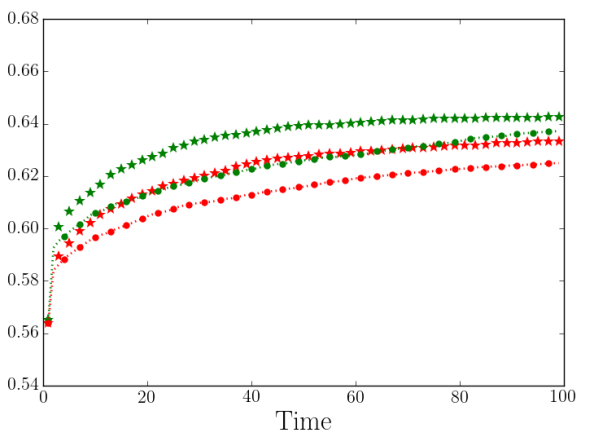

(a) $K=1, C=1$

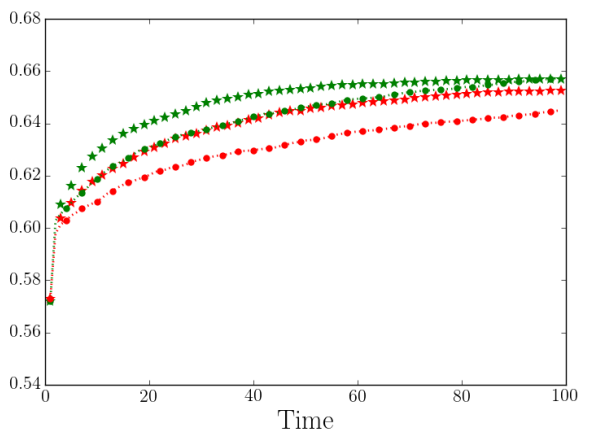

(c) $K=3, C=1$

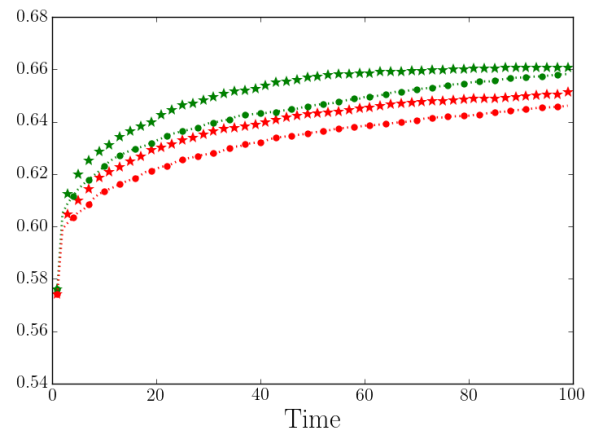

(b) $K=1, C=3$

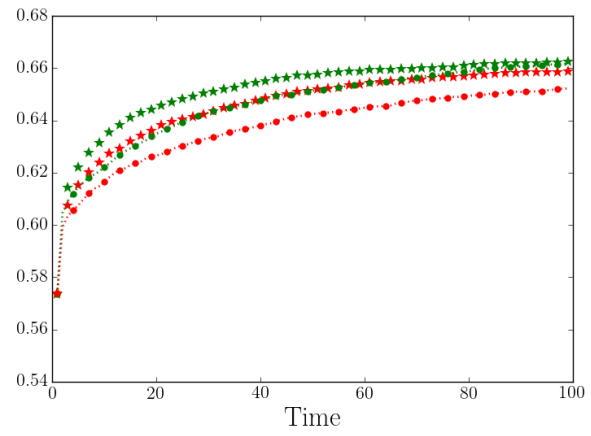

(d) $K=3, C=3$

Figure 17 The best quality performance of PD projects with diverse teams ( developing peripheral $(* * * * * * * * *)$. Subsystems interact as modular (blue), cyclical (green), and acyclical (red) patterns. Each point is average performance of 100 simulation runs, and $n_{s}=n_{q}=5, n_{e}=4$. 


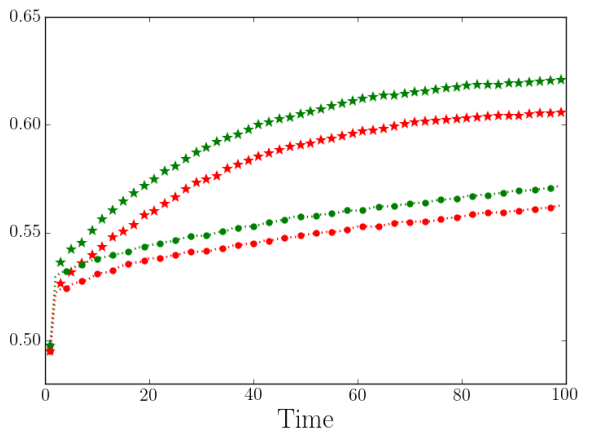

(a) $K=1, C=1$

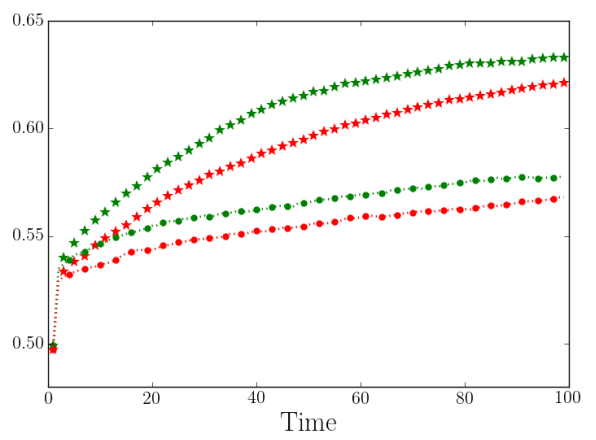

(c) $K=3, C=1$

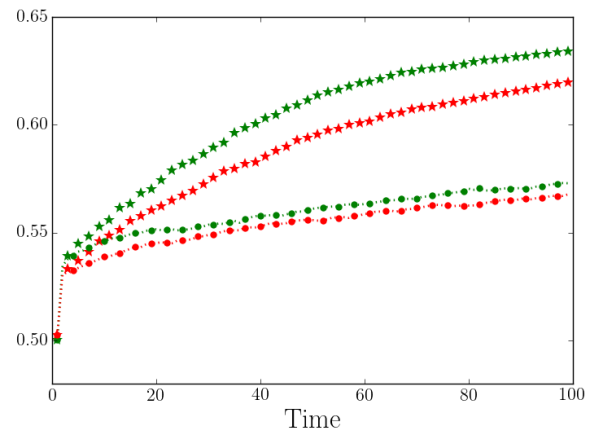

(b) $K=1, C=3$

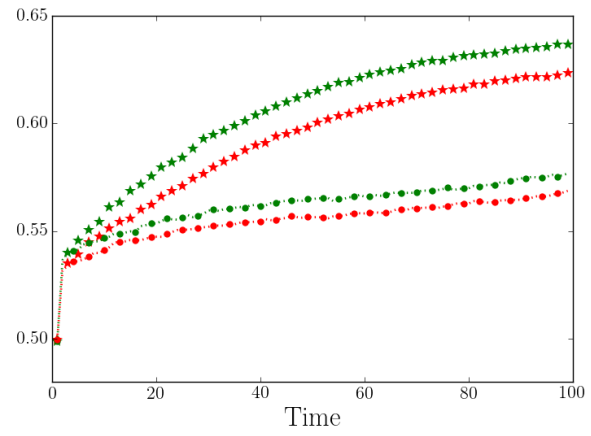

(d) $K=3, C=3$

Figure 18 The average quality performance of PD projects with diverse teams (-.-.-.-.-.-๑) and diverse teams developing peripheral (*********). Subsystems interact as modular (blue), cyclical (green), and acyclical (red) patterns. Each point is average performance of 100 simulation runs, and $n_{s}=n_{q}=5, n_{e}=4$. 


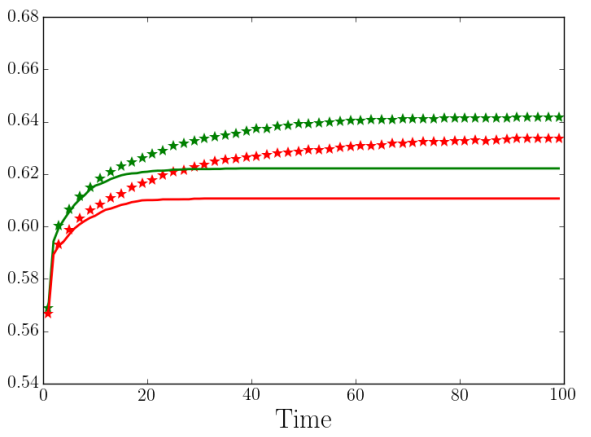

(a) $K=1, C=1$

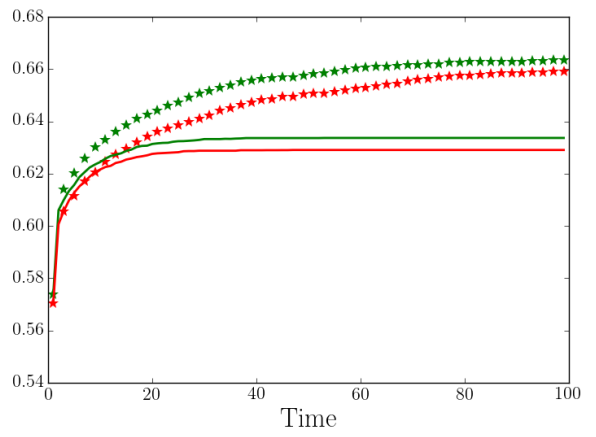

(c) $K=3, C=1$

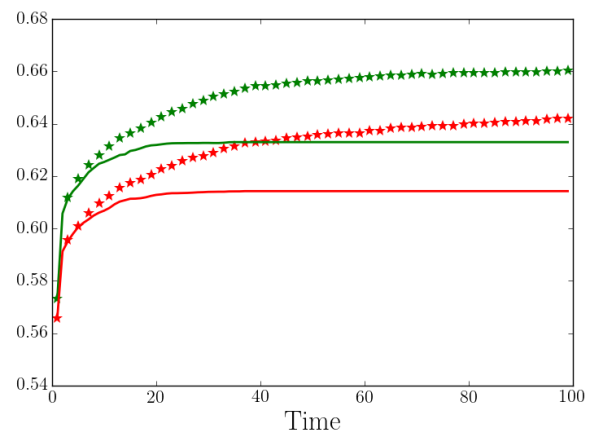

(b) $K=1, C=3$

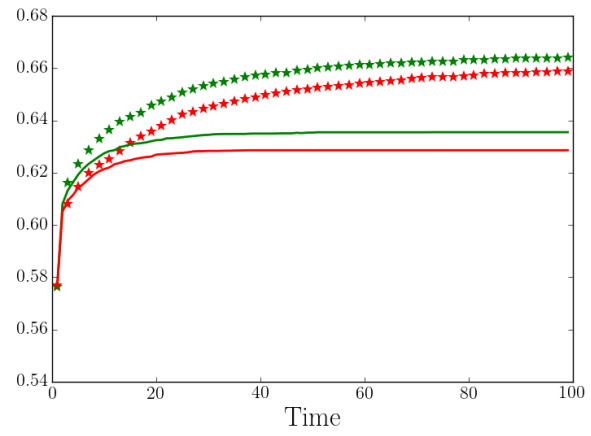

(d) $K=3, C=3$

Figure 19 The best quality performance of PD projects with communicative teams ( $\longrightarrow$ ) and diverse teams developing peripheral (*********). Subsystems interact as modular (blue), cyclical (green), and acyclical (red) patterns. Each point is average performance of 100 simulation runs, and $n_{s}=n_{q}=5, n_{e}=4$. 


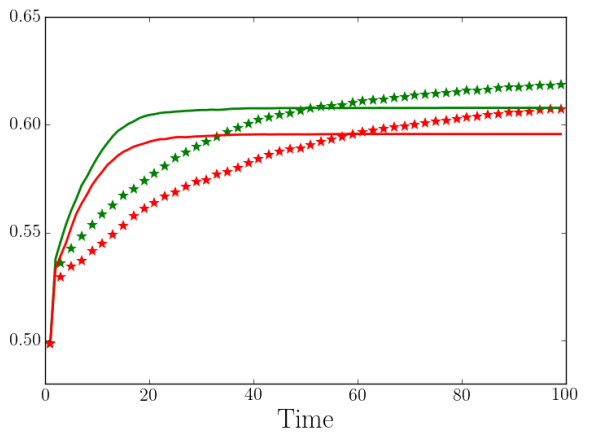

(a) $K=1, C=1$

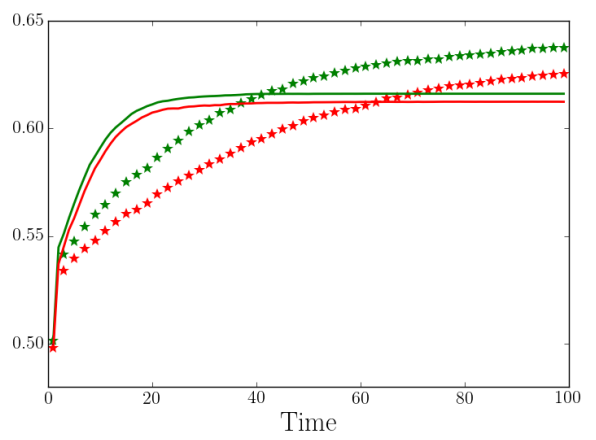

(c) $K=3, C=1$

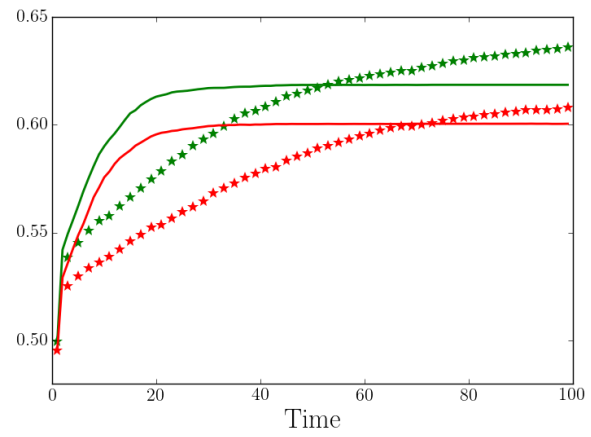

(b) $K=1, C=3$

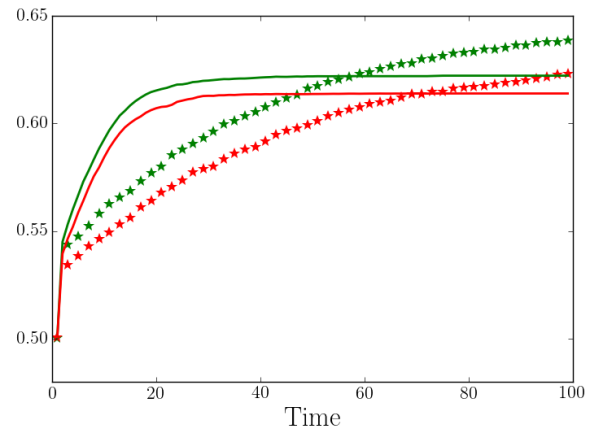

(d) $K=3, C=3$

Figure 20 The average quality performance of PD projects with communicative teams ( - ) and diverse teams developing peripheral $(* * * * * * * * *)$. Subsystems interact as modular (blue), cyclical (green), and acyclical (red) patterns. Each point is average performance of $\mathbf{1 0 0}$ simulation runs, and $n_{s}=n_{q}=5, n_{e}=4$. 


\section{Experiment 2: Tables}

Table 9 Paired t-tests comparing the best quality performance of PD systems with diverse teams developing core and diverse teams; For these PD systems, $n_{s}=n_{q}=5$ and $n_{e}=4$.

\begin{tabular}{|c|c|c|c|c|c|c|}
\hline \multicolumn{3}{|c|}{ Inputs } & \multirow{2}{*}{ Pattern } & \multirow{2}{*}{ t-value } & \multirow{2}{*}{ Pattern } & \multirow{2}{*}{ t-value } \\
\hline$n_{q}$ & $K$ & $C$ & & & & \\
\hline \multirow{4}{*}{5} & 1 & 1 & \multirow{8}{*}{ Cyclical } & $3.287^{*}$ & \multirow{8}{*}{ Acyclical } & $4.21^{*}$ \\
\hline & 1 & 2 & & -0.156 & & 1.662 \\
\hline & 2 & 1 & & 1.227 & & $2.803^{*}$ \\
\hline & 2 & 2 & & 0.034 & & 1.625 \\
\hline \multirow{4}{*}{7} & 1 & 1 & & $3.816^{*}$ & & $5.308^{*}$ \\
\hline & 1 & 2 & & 1.617 & & 2.087 \\
\hline & 2 & 1 & & $3.373^{*}$ & & $4.014^{*}$ \\
\hline & 2 & 2 & & 1.265 & & 2.265 \\
\hline
\end{tabular}

Table 10 Paired t-tests comparing the best quality performance of PD systems with diverse teams developing core and communicative teams; For these PD systems, $n_{s}=n_{q}=5$ and $n_{e}=4$.

\begin{tabular}{|c|c|c|c|c|c|c|}
\hline \multicolumn{3}{|c|}{ Inputs } & \multirow{2}{*}{ Pattern } & \multirow{2}{*}{ t-value } & \multirow{2}{*}{ Pattern } & \multirow{2}{*}{ t-value } \\
\hline$n_{q}$ & $K$ & $C$ & & & & \\
\hline \multirow{4}{*}{5} & 1 & 1 & \multirow{8}{*}{ Cyclical } & $8.925^{*}$ & \multirow{8}{*}{ Acyclical } & $11.697^{*}$ \\
\hline & 1 & 2 & & $11.252^{*}$ & & $12.507^{*}$ \\
\hline & 2 & 1 & & $11.374^{*}$ & & $12.795^{*}$ \\
\hline & 2 & 2 & & $13.106^{*}$ & & $13.676^{*}$ \\
\hline \multirow{4}{*}{7} & 1 & 1 & & $12.909^{*}$ & & $10.89^{*}$ \\
\hline & 1 & 2 & & $12.991^{*}$ & & $10.447^{*}$ \\
\hline & 2 & 1 & & $10.415^{*}$ & & $11.88^{*}$ \\
\hline & 2 & 2 & & $11.478^{*}$ & & $10.656^{*}$ \\
\hline
\end{tabular}

Table 11 Paired t-tests comparing the best quality performance of PD systems with diverse teams developing peripheral and diverse teams; For these PD systems, $n_{s}=n_{q}=5$ and $n_{e}=4$.

\begin{tabular}{|c|c|c|c|c|c|c|}
\hline \multicolumn{3}{|c|}{ Inputs } & \multirow{2}{*}{ Pattern } & \multirow{2}{*}{ t-value } & \multirow{2}{*}{ Pattern } & \multirow{2}{*}{ t-value } \\
\hline$n_{q}$ & $K$ & $C$ & & & & \\
\hline \multirow{4}{*}{5} & 1 & 1 & \multirow{8}{*}{ Cyclical } & $2.968^{*}$ & \multirow{8}{*}{ Acyclical } & $5.085^{*}$ \\
\hline & 1 & 2 & & 1.62 & & 2.532 \\
\hline & 2 & 1 & & -0.034 & & $4.289^{*}$ \\
\hline & 2 & 2 & & 0.491 & & $3.873^{*}$ \\
\hline \multirow{4}{*}{7} & 1 & 1 & & $6.909^{*}$ & & $5.757^{*}$ \\
\hline & 1 & 2 & & 2.091 & & $5.365^{*}$ \\
\hline & 2 & 1 & & $3.351^{*}$ & & $7.689^{*}$ \\
\hline & 2 & 2 & & 2.511 & & $5.187^{*}$ \\
\hline
\end{tabular}


Table 12 Paired t-tests comparing the best quality performance of PD systems with diverse teams developing peripheral and communicative teams; For these PD systems, $n_{s}=n_{q}=5$ and $n_{e}=4$.

\begin{tabular}{|c|c|c|c|c|c|c|}
\hline \multicolumn{3}{|c|}{ Inputs } & \multirow{2}{*}{ Pattern } & \multirow{2}{*}{ t-value } & \multirow{2}{*}{ Pattern } & \multirow{2}{*}{ t-value } \\
\hline$n_{q}$ & $K$ & $C$ & & & & \\
\hline \multirow{4}{*}{5} & 1 & 1 & \multirow{8}{*}{ Cyclical } & $9.852^{*}$ & \multirow{8}{*}{ Acyclical } & $12.78^{*}$ \\
\hline & 1 & 2 & & $11.84^{*}$ & & $15.586^{*}$ \\
\hline & 2 & 1 & & $12.92^{*}$ & & $12.629^{*}$ \\
\hline & 2 & 2 & & $15.036^{*}$ & & $14.591^{*}$ \\
\hline \multirow{4}{*}{7} & 1 & 1 & & $12.605^{*}$ & & $10.937^{*}$ \\
\hline & 1 & 2 & & $12.53^{*}$ & & $12.597^{*}$ \\
\hline & 2 & 1 & & $12.885^{*}$ & & $13.633^{*}$ \\
\hline & 2 & 2 & & $14.02^{*}$ & & $14.198^{*}$ \\
\hline
\end{tabular}

Table 13 Paired t-tests comparing the average quality performance of PD systems with with diverse teams developing core and diverse teams; For these PD systems, $n_{s}=n_{q}=5$ and $n_{e}=4$.

\begin{tabular}{|c|c|c|c|c|c|c|}
\hline \multicolumn{3}{|c|}{ Inputs } & \multirow{2}{*}{ Pattern } & \multirow{2}{*}{ t-value } & \multirow{2}{*}{ Pattern } & \multirow{2}{*}{ t-value } \\
\hline$n_{q}$ & $K$ & $C$ & & & & \\
\hline \multirow{4}{*}{5} & 1 & 1 & \multirow{8}{*}{ Cyclical } & $19.081^{*}$ & \multirow{8}{*}{ Acyclical } & $20.341^{*}$ \\
\hline & 1 & 2 & & $21.443^{*}$ & & $22.414^{*}$ \\
\hline & 2 & 1 & & $19.516^{*}$ & & $21.586^{*}$ \\
\hline & 2 & 2 & & $23.698^{*}$ & & $25.975^{*}$ \\
\hline \multirow{4}{*}{7} & 1 & 1 & & $23.934^{*}$ & & $31.592^{*}$ \\
\hline & 1 & 2 & & $32.815^{*}$ & & $25.529^{*}$ \\
\hline & 2 & 1 & & $24.05^{*}$ & & $35.061^{*}$ \\
\hline & 2 & 2 & & $32.018^{*}$ & & $27.347^{*}$ \\
\hline
\end{tabular}

Table 14 Paired t-tests comparing the average quality performance of PD systems with diverse teams developing core and communicative teams; For these PD systems, $n_{s}=n_{q}=5$ and $n_{e}=4$.

\begin{tabular}{|c|c|c|c|c|c|c|}
\hline \multicolumn{3}{|c|}{ Inputs } & \multirow{2}{*}{ Pattern } & \multirow{2}{*}{ t-value } & \multirow{2}{*}{ Pattern } & \multirow{2}{*}{ t-value } \\
\hline$n_{q}$ & $K$ & $C$ & & & & \\
\hline \multirow{4}{*}{5} & 1 & 1 & \multirow{8}{*}{ Cyclical } & $3.052^{*}$ & \multirow{8}{*}{ Acyclical } & $7.861^{*}$ \\
\hline & 1 & 2 & & $4.363^{*}$ & & $6.879^{*}$ \\
\hline & 2 & 1 & & $5.227^{*}$ & & $8.654^{*}$ \\
\hline & 2 & 2 & & $5.07^{*}$ & & $6.451^{*}$ \\
\hline \multirow{4}{*}{7} & 1 & 1 & & -0.826 & & 1.091 \\
\hline & 1 & 2 & & -2.296 & & -1.572 \\
\hline & 2 & 1 & & -1.09 & & 0.737 \\
\hline & 2 & 2 & & $-3.926^{*}$ & & -0.871 \\
\hline
\end{tabular}

Table 15 Paired t-tests comparing the average quality performance of PD systems with diverse teams developing peripheral and diverse teams; For these PD systems, $n_{s}=n_{q}=5$ and $n_{e}=4$.

\begin{tabular}{|c|c|c|c|c|c|c|}
\hline \multicolumn{3}{|c|}{ Inputs } & \multirow{2}{*}{ Pattern } & \multirow{2}{*}{ t-value } & \multirow{2}{*}{ Pattern } & \multirow{2}{*}{ t-value } \\
\hline$n_{q}$ & $K$ & $C$ & & & & \\
\hline \multirow{4}{*}{5} & 1 & 1 & \multirow{8}{*}{ Cyclical } & $21.828^{*}$ & \multirow{8}{*}{ Acyclical } & $20.15^{*}$ \\
\hline & 1 & 2 & & $23.395^{*}$ & & $22.851^{*}$ \\
\hline & 2 & 1 & & $23.688^{*}$ & & $18.973^{*}$ \\
\hline & 2 & 2 & & $27.923^{*}$ & & $23.154^{*}$ \\
\hline \multirow{4}{*}{7} & 1 & 1 & & $24.459^{*}$ & & $16.743^{*}$ \\
\hline & 1 & 2 & & $27.422^{*}$ & & $20.899^{*}$ \\
\hline & 2 & 1 & & $26.708^{*}$ & & $22.105^{*}$ \\
\hline & 2 & 2 & & $27.385^{*}$ & & $22.327^{*}$ \\
\hline
\end{tabular}


Table 16 Paired t-tests comparing the average quality performance of PD systems with diverse teams developing peripheral and communicative teams; For these PD systems, $n_{s}=n_{q}=5$ and $n_{e}=4$.

\begin{tabular}{|c|c|c|c|c|c|c|}
\hline \multicolumn{3}{|c|}{ Inputs } & \multirow{2}{*}{ Pattern } & \multirow{2}{*}{ t-value } & \multirow{2}{*}{ Pattern } & \multirow{2}{*}{ t-value } \\
\hline$n_{q}$ & $K$ & $C$ & & & & \\
\hline \multirow{4}{*}{5} & 1 & 1 & \multirow{8}{*}{ Cyclical } & $4.331^{*}$ & \multirow{8}{*}{ Acyclical } & $5.737^{*}$ \\
\hline & 1 & 2 & & $6.392^{*}$ & & $3.015^{*}$ \\
\hline & 2 & 1 & & $8.147^{*}$ & & $5.017^{*}$ \\
\hline & 2 & 2 & & $7.409^{*}$ & & $3.183^{*}$ \\
\hline \multirow{4}{*}{7} & 1 & 1 & & $-3.42^{*}$ & & $-5.452^{*}$ \\
\hline & 1 & 2 & & -1.87 & & $-7.148^{*}$ \\
\hline & 2 & 1 & & -2.057 & & $-6.42^{*}$ \\
\hline & 2 & 2 & & $-3.314^{*}$ & & $-6.838^{*}$ \\
\hline
\end{tabular}




\section{Experiment 2: Tables- $\mathrm{W}=2$ Setting}

Table 17 Paired t-tests comparing the best quality performance of PD systems with diverse teams developing core and diverse teams; For these PD systems, $n_{s}=n_{q}=5$ and $n_{e}=4$.

\begin{tabular}{|c|c|c|c|c|c|c|}
\hline \multicolumn{3}{|c|}{ Inputs } & \multirow{2}{*}{ Pattern } & \multirow{2}{*}{ t-value } & \multirow{2}{*}{ Pattern } & \multirow{2}{*}{ t-value } \\
\hline$n_{q}$ & $K$ & $C$ & & & & \\
\hline \multirow{4}{*}{5} & 1 & 1 & \multirow{8}{*}{ Cyclical } & 1.188 & \multirow{8}{*}{ Acyclical } & $2.848^{* * *}$ \\
\hline & 1 & 2 & & -2.154 & & 0.406 \\
\hline & 2 & 1 & & -0.435 & & $3.48^{* * *}$ \\
\hline & 2 & 2 & & -1.334 & & -0.962 \\
\hline \multirow{4}{*}{7} & 1 & 1 & & 1.938 & & $5.126^{* * *}$ \\
\hline & 1 & 2 & & 0.834 & & 0.286 \\
\hline & 2 & 1 & & $3.338^{* * *}$ & & $4.347^{* * *}$ \\
\hline & 2 & 2 & & -1.307 & & 1.618 \\
\hline
\end{tabular}

Table 18 Paired t-tests comparing the best quality performance of PD systems with diverse teams developing core and communicative teams; For these PD systems, $n_{s}=n_{q}=5$ and $n_{e}=4$.

\begin{tabular}{|c|c|c|c|c|c|c|}
\hline \multicolumn{3}{|c|}{ Inputs } & \multirow{2}{*}{ Pattern } & \multirow{2}{*}{ t-value } & \multirow{2}{*}{ Pattern } & \multirow{2}{*}{ t-value } \\
\hline$n_{q}$ & $K$ & $C$ & & & & \\
\hline \multirow{4}{*}{5} & 1 & 1 & \multirow{8}{*}{ Cyclical } & $10.306^{* * *}$ & \multirow{8}{*}{ Acyclical } & $9.762^{* * *}$ \\
\hline & 1 & 2 & & $12.23^{* * *}$ & & $10.718^{* * *}$ \\
\hline & 2 & 1 & & $10.344^{* * *}$ & & $11.939^{* * *}$ \\
\hline & 2 & 2 & & $8.957^{* * *}$ & & $11.629^{* * *}$ \\
\hline \multirow{4}{*}{7} & 1 & 1 & & $8.551^{* * *}$ & & $11.065^{* * *}$ \\
\hline & 1 & 2 & & $8.629^{* * *}$ & & $10.124^{* * *}$ \\
\hline & 2 & 1 & & $8.874^{* * *}$ & & $11.824^{* * *}$ \\
\hline & 2 & 2 & & $10.857^{* * *}$ & & $10.984^{* * *}$ \\
\hline
\end{tabular}

Table 19 Paired t-tests comparing the best quality performance of PD systems with diverse teams developing peripheral and diverse teams; For these PD systems, $n_{s}=n_{q}=5$ and $n_{e}=4$.

\begin{tabular}{|c|c|c|c|c|c|c|}
\hline \multicolumn{3}{|c|}{ Inputs } & \multirow{2}{*}{ Pattern } & \multirow{2}{*}{ t-value } & \multirow{2}{*}{ Pattern } & \multirow{2}{*}{ t-value } \\
\hline$n_{q}$ & $K$ & $C$ & & & & \\
\hline \multirow{4}{*}{5} & 1 & 1 & \multirow{8}{*}{ Cyclical } & 2.619 & \multirow{8}{*}{ Acyclical } & $7.453^{* * *}$ \\
\hline & 1 & 2 & & 0.045 & & 2.513 \\
\hline & 2 & 1 & & 2.524 & & $4.8^{* * *}$ \\
\hline & 2 & 2 & & $-2.931^{* * *}$ & & 0.811 \\
\hline \multirow{4}{*}{7} & 1 & 1 & & $5.812^{* * *}$ & & $5.817^{* * *}$ \\
\hline & 1 & 2 & & 1.541 & & $5.353^{* * *}$ \\
\hline & 2 & 1 & & $3.962^{* * *}$ & & $5.148^{* * *}$ \\
\hline & 2 & 2 & & 1.457 & & $4.215^{* * *}$ \\
\hline
\end{tabular}


Table 20 Paired t-tests comparing the best quality performance of PD systems with diverse teams developing peripheral and communicative teams; For these PD systems, $n_{s}=n_{q}=5$ and $n_{e}=4$.

\begin{tabular}{|c|c|c|c|c|c|c|}
\hline \multicolumn{3}{|c|}{ Inputs } & \multirow{2}{*}{ Pattern } & \multirow{2}{*}{ t-value } & \multirow{2}{*}{ Pattern } & \multirow{2}{*}{ t-value } \\
\hline$n_{q}$ & $K$ & $C$ & & & & \\
\hline \multirow{4}{*}{5} & 1 & 1 & \multirow{8}{*}{ Cyclical } & $9.606^{* * *}$ & \multirow{8}{*}{ Acyclical } & $12.765^{* * *}$ \\
\hline & 1 & 2 & & $11.043^{* * *}$ & & $14.091^{* * *}$ \\
\hline & 2 & 1 & & $11.909^{* * *}$ & & $12.85^{* * *}$ \\
\hline & 2 & 2 & & $10.601^{* * *}$ & & $13.372^{* * *}$ \\
\hline \multirow{4}{*}{7} & 1 & 1 & & $9.731^{* * *}$ & & $13.663^{* * *}$ \\
\hline & 1 & 2 & & $9.811^{* * *}$ & & $11.479^{* * *}$ \\
\hline & 2 & 1 & & $10.446^{* * *}$ & & $15.793^{* * *}$ \\
\hline & 2 & 2 & & $11.615^{* * *}$ & & $13.665^{* * *}$ \\
\hline
\end{tabular}

Table 21 Paired t-tests comparing the average quality performance of PD systems with with diverse teams developing core and diverse teams; For these PD systems, $n_{s}=n_{q}=5$ and $n_{e}=4$.

\begin{tabular}{|c|c|c|c|c|c|c|}
\hline \multicolumn{3}{|c|}{ Inputs } & \multirow{2}{*}{ Pattern } & \multirow{2}{*}{ t-value } & \multirow{2}{*}{ Pattern } & \multirow{2}{*}{ t-value } \\
\hline$n_{q}$ & $K$ & $C$ & & & & \\
\hline \multirow{4}{*}{5} & 1 & 1 & \multirow{8}{*}{ Cyclical } & $19.121^{\text {*** }}$ & \multirow{8}{*}{ Acyclical } & $19.649^{* * *}$ \\
\hline & 1 & 2 & & $20.621^{* * *}$ & & $19.813^{* * *}$ \\
\hline & 2 & 1 & & $19.539^{* * *}$ & & $24.784^{* * *}$ \\
\hline & 2 & 2 & & $23.689^{* * *}$ & & $23.123^{* * *}$ \\
\hline \multirow{4}{*}{7} & 1 & 1 & & $23.743^{* * *}$ & & $27.418^{* * *}$ \\
\hline & 1 & 2 & & $27.062^{* * *}$ & & $29.845^{* * *}$ \\
\hline & 2 & 1 & & $26.82^{* * *}$ & & $31.368^{* * *}$ \\
\hline & 2 & 2 & & $31.225^{\text {*** }}$ & & $35.903^{* * *}$ \\
\hline
\end{tabular}

Table 22 Paired t-tests comparing the average quality performance of PD systems with diverse teams developing core and communicative teams; For these PD systems, $n_{s}=n_{q}=5$ and $n_{e}=4$.

\begin{tabular}{|c|c|c|c|c|c|c|}
\hline \multicolumn{3}{|c|}{ Inputs } & \multirow{2}{*}{ Pattern } & \multirow{2}{*}{ t-value } & \multirow{2}{*}{ Pattern } & \multirow{2}{*}{ t-value } \\
\hline$n_{q}$ & $K$ & $C$ & & & & \\
\hline \multirow{4}{*}{5} & 1 & 1 & \multirow{8}{*}{ Cyclical } & $4.056^{* * *}$ & \multirow{8}{*}{ Acyclical } & $9.285^{* * *}$ \\
\hline & 1 & 2 & & $4.753^{* * *}$ & & $6.99^{* * *}$ \\
\hline & 2 & 1 & & $4.796^{* * *}$ & & $9.248^{* * *}$ \\
\hline & 2 & 2 & & $4.953^{* * *}$ & & $10.625^{* * *}$ \\
\hline \multirow{4}{*}{7} & 1 & 1 & & $-3.353^{* * *}$ & & $2.885^{* * *}$ \\
\hline & 1 & 2 & & -0.515 & & $4.267^{* * *}$ \\
\hline & 2 & 1 & & -2.08 & & $6.978^{* * *}$ \\
\hline & 2 & 2 & & 0.483 & & $6.011^{* * *}$ \\
\hline
\end{tabular}

Table 23 Paired t-tests comparing the average quality performance of PD systems with diverse teams developing peripheral and diverse teams; For these PD systems, $n_{s}=n_{q}=5$ and $n_{e}=4$.

\begin{tabular}{|c|c|c|c|c|c|c|}
\hline \multicolumn{3}{|c|}{ Inputs } & \multirow{2}{*}{ Pattern } & \multirow{2}{*}{ t-value } & \multirow{2}{*}{ Pattern } & \multirow{2}{*}{ t-value } \\
\hline$n_{q}$ & $K$ & $C$ & & & & \\
\hline \multirow{4}{*}{5} & 1 & 1 & \multirow{8}{*}{ Cyclical } & $15.032^{* * *}$ & \multirow{8}{*}{ Acyclical } & $19.827^{* * *}$ \\
\hline & 1 & 2 & & $20.404^{* * *}$ & & $24.322^{* * *}$ \\
\hline & 2 & 1 & & $22.694^{* * *}$ & & $22.733^{* * *}$ \\
\hline & 2 & 2 & & $22.035^{* * *}$ & & $22.544^{* * *}$ \\
\hline \multirow{4}{*}{7} & 1 & 1 & & $22.686^{* * *}$ & & $19.512^{* * *}$ \\
\hline & 1 & 2 & & $25.369^{* * *}$ & & $22.466^{* * *}$ \\
\hline & 2 & 1 & & $23.111^{* * *}$ & & $19.743^{* * *}$ \\
\hline & 2 & 2 & & $30.427^{* * *}$ & & $26.921^{* * *}$ \\
\hline
\end{tabular}


Table 24 Paired t-tests comparing the average quality performance of PD systems with diverse teams developing peripheral and communicative teams; For these PD systems, $n_{s}=n_{q}=5$ and $n_{e}=4$.

\begin{tabular}{|c|c|c|c|c|c|c|}
\hline \multicolumn{3}{|c|}{ Inputs } & \multirow{2}{*}{ Pattern } & \multirow{2}{*}{ t-value } & \multirow{2}{*}{ Pattern } & \multirow{2}{*}{ t-value } \\
\hline$n_{q}$ & $K$ & $C$ & & & & \\
\hline \multirow{4}{*}{5} & 1 & 1 & \multirow{8}{*}{ Cyclical } & $3.165^{* * *}$ & \multirow{8}{*}{ Acyclical } & $6.521^{* * *}$ \\
\hline & 1 & 2 & & $4.121^{* * *}$ & & $8.235^{* * *}$ \\
\hline & 2 & 1 & & $4.643^{* * *}$ & & $5.125^{* * *}$ \\
\hline & 2 & 2 & & $4.747^{* * *}$ & & $8.589^{* * *}$ \\
\hline \multirow{4}{*}{7} & 1 & 1 & & $-3.257^{* * *}$ & & $-4.471^{* * *}$ \\
\hline & 1 & 2 & & -2.178 & & -1.44 \\
\hline & 2 & 1 & & $-3.293^{* * *}$ & & -1.636 \\
\hline & 2 & 2 & & -1.397 & & 0.572 \\
\hline
\end{tabular}

